\title{
THE LATERAL COMPLETION OF AN ARBITRARY LATTICE GROUP
}

\author{
S. J. BERNAU \\ (Received 16 January 1973) \\ Communicated by G. E. Wall
}

\begin{abstract}
SUMMARY
This paper shows that every lattice group $G$ can be densely embedded in a unique laterally complete lattice group $H$ (the lateral completion of $G$ ). All reasonable structure properties of $G$ are inherited by $H$ and we have the following relationships between the ideal radical $L(G)$, the distributive radical $D(G)$ and the radical $R(G)$ of $G$ and the corresponding radicals of $H$.
\end{abstract}

$$
L(G) \cup(L(H) \cap G) \subset D(H) \cap G=D(G) \subset R(H) \cap G=R(G)
$$

\section{Introduction}

We first recall some definitions. Let $G$ be a lattice group. A positive disjoint subset of $G$ is a non-empty subset $M$ of $G^{+}(=\{x \in G: x \geqq 0\})$ such that $m \wedge n=0$ if $m, n \in M$ and $m \neq n$, i.e., the elements of $M$ are pairwise disjoint; $G$ is laterally complete if every positive disjoint subset of $G$ has a supremum. Conrad (1969) defines an $\mathscr{L}$-completion of $G$ as follows. If $G$ is a sublattice group of $H$ and if, for each positive disjoint subset $M$ of $G$ such that $V_{G} M$ exists, then $\bigvee_{H} M$ exists and is equal to $\bigvee_{G} M$, then $G$ is an $\mathscr{L}$-subgroup of $H$. If $H$ is laterally complete, $G$ is an $\mathscr{L}$-subgroup of $H$, and $K$ is the intersection of all laterally complete $\mathscr{L}$-subgroups of $H$ that contain $G$ then $K$ is called an $\mathscr{L}$ completion of $G$ or the $\mathscr{L}$-completion of $G$ in $H ; K$ is laterally complete and no proper $\mathscr{L}$-subgroup of $K$ is laterally complete and contains $G$.

As Conrad points out $\mathscr{L}$-completions can be rather pathological. However, if $G$ is dense in an $\mathscr{L}$-completion $K$, i.e., for each non-zero $k \in K^{+}$there exists a non-zero $g \in G$ such that $0<g \leqq k$, all the pathology disappears. He is then led to the following definition. A lateral completion of $G$ is a lattice group $K \supset G$ such that:

(I) $G$ is dense in $K$;

(II) $K$ is laterally complete;

(III) no proper sublattice group of $K$ contains $G$ and is laterally complete. (Here we do not distinguish genuine inclusion from embedding.) 
The existence of a unique lateral completion for a complete lattice group goes back to Nakano (1950) and Pinsker (1949). The author (1966) introduced the concept of an orthocomplete lattice group which is laterally complete and in which each polar subgroup is a direct summand.

An orthocompletion of $G$ was then defined to be a laterally complete group $H$ which contains $G$ as a sublattice group and in which every positive element has a representation in terms of elements of $G$ and polars of subsets of $G$. It was shown that a necessary and sufficient condition for the existence of an orthocompletion of $G$ is that $G$ be representable (see Section 4 for this definition). This construction was based on methods of Nakano (1950) and Amemiya (1953).

Conrad (1969) gives a much simpler construction for the orthocompletion using the concept of a direct limit of lattice groups, and uses this to derive the existence of a unique lateral completion for a representable lattice group. He also gives a quite different construction of a unique lateral completion (with zero radical) for lattice groups with zero radical (see Section 5 for definitions). Byrd and Lloyd (1969) show that Conrad's construction can be applied to lattice groups whose distributive radical is zero, and gives a unique lateral completion with zero distributive radical.

Conrad's construction can be summarised as follows. The Holland representation, Holland (1963), for a lattice group shows the existence of $\mathscr{L}$-completions (but not uniqueness). Conrad chooses a suitably constructed $\mathscr{L}$-completion $K$ for a lattice group $G$ and defines, for any subset $X$ of $K, X$ to be the sublattice group of $K$ generated by all elements of the form $\vee M$ with $M$ a positive disjoint subset of $X$. Then, with $G(0)=G$, for each ordinal $\mu$ he defines

$$
\begin{array}{ll}
G(\mu)=\overline{G(v)} & \text { if } \mu=v+1 \\
G(\mu)=\bigcup_{\nu<\mu} G(v) & \text { if } \mu \text { is a limit ordinal. }
\end{array}
$$

The process terminates for some least ordinal $\lambda$ and $K=G(\lambda)$. Results about $K$ are then largely proved by transfinite induction. In most cases only $G(1)$ needs to be considered, the remaining steps of the proof being entirely straightforward.

Our construction may now be outlined. In Section 2 we produce an external extension $\bar{G}$ of $G$ in which $G$ is dense and every positive disjoint subset of $G$ has a supremum. The elements of $\bar{G}$ are equivalence classes in a semigroup manufactured from positive disjoint subsets of $G, g \in G^{+}$corresponds to the equivalence class of $\{g\}$ in $\bar{G}$, and the equivalence class determined by a positive disjoint subset of $G$ becomes the supremum of the corresponding subset of $\bar{G}$. The equivalence relation is so chosen that (the image of) $G$ is dense in $\bar{G}$. In section 3 we define $G(\mu)=\overline{G(v)}$ when $\mu=v+1$ and for limit ordinals use a simple direct limit in place of the set-theoretic union of Conrad's construction. This time the fact that $G$ is dense in each $G(\mu)$ puts a limit on the cardinality of positive disjoint subsets 
and enables us to prove that the construction terminates, i.e., that $G(\mu+1)$ $=G(\mu)$, for some ordinal $\mu$. By choosing the least such ordinal, $\lambda$ say, we find that $G(\lambda)$ is a lateral completion of $G$. The method of construction enables an easy proof of uniqueness to be given.

The choice of notation in sections 2,3 is deliberately intended to parallel Conrad's notation. This enables us later to use, where applicable, the same arguments for $\mathscr{L}$-completions and lateral completions. The only distinguishing feature is the denseness of $G$ in its lateral completion.

Verification of certain structure properties of the lateral completion, in section 4 , is routine. In section 5 we investigate the relationship between the radicals of $G$ and those of an $\mathscr{L}$-completion $K$ of $G$. We extend some of Conrad's results and get particularly sharp results when $K$ is the lateral completion of $G$. In section 6 we give a very simple proof of one Conrad's results (1969), Theorem 6.1 about the $\mathscr{L}$-completion of a lattice group satisfying the condition that each upper bounded positive disjoint subset is finite.

I am grateful to Paul Conrad for supplying a preprint of Conrad (1969) and to Richard Byrd for discovering non-trivial errors in the first version of section 2 and in my first efforts at correction. Yet another error, in the proof of Lemma 2.3, was discovered by the referee of the second revision. Roger Bleier sat through a detailed seminar presentation of this material. His refusal to be bluffed and his insistence on following all steps of the proofs led to many improvements in the exposition.

\section{The basic extension}

Let $G$ be a lattice group and $M$ and $N$ any non-empty subsets of $G$. We use a natural notation and write $M \pm N, M \vee N, M \wedge N$, for the subsets of $G$ consisting respectively of all elements of $G$ of the form $m \pm n, m \vee n, m \wedge n$, for all $m \in M, n \in N$. The natural extension of this notation to the case of finite index sets $A, B_{\alpha}(\alpha \in A)$ and subsets $M_{\alpha \beta}$ of $G$ will be used to define $\bigvee_{\alpha \in A} \wedge_{\beta \in B_{\alpha}} M_{\alpha \beta}$.

Now let $\mathscr{D}$ denote the collection of all positive disjoint subsets of $G$. Define a semigroup $\mathscr{H}$ as follows. The elements of $\mathscr{H}$ are ordered $n$-tuples $(n=1,2, \cdots)$ of the form $\left(\varepsilon_{1} M_{1}, \cdots, \varepsilon_{n} M_{n}\right)$ where $\varepsilon_{i}= \pm 1$ and $M_{i} \in \mathscr{D}(i=1, \cdots, n)$. We define

$$
\left(\varepsilon_{1} M_{1}, \cdots, \varepsilon_{n} M_{n}\right)+\left(\eta_{1} N_{1}, \cdots, \eta_{k} N_{k}\right)=\left(\varepsilon_{1} M_{1}, \cdots, \varepsilon_{n} M_{n}, \eta_{1} N_{1}, \cdots, \eta_{k} N_{k}\right) .
$$

This makes $\mathscr{H}$ an associative semigroup under addition. Further we define, for $H=\left(\varepsilon_{1} M_{1}, \cdots, \varepsilon_{n} M_{n}\right) \in \mathscr{H},-H=\left(-\varepsilon_{n} M_{n}, \cdots,-\varepsilon_{1} M_{1}\right)$ and, for $H_{1}, H_{2} \in \mathscr{H}$, $H_{1}-H_{2}=H_{1}+\left(-H_{2}\right),-H_{1}+H_{2}=\left(-H_{1}\right)+H_{2}$. For each

$$
H=\left(\varepsilon_{1} M_{1}, \cdots, \varepsilon_{n} M_{n}\right) \in \mathscr{H}
$$

we define $\bar{H} \subset G$ by $\bar{H}=\varepsilon_{1} M_{1}+\cdots+\varepsilon_{n} M_{n}$. Finally let $\mathscr{G}$ denote the set of all non-empty finite sets of non-empty finite subsets of $\mathscr{H}$. In other words a typical element $L$ of $\mathscr{G}$ is of the form $L=\left\{\left\{H_{\alpha \beta}: \beta \in B_{\alpha}\right\}: \alpha \in A\right\}$ where $A$ 
and $B_{\alpha}(\alpha \in A)$ are non-empty finite index sets. For such an element $L$ we shall write $L_{\alpha}=\left\{H_{\alpha \beta}: \beta \in B_{\alpha}\right\}(\alpha \in A)$, we may also write $L=\left\{\left\{H_{\alpha \beta}\right\}\right\}$, supressing index sets, where no confusion is likely. For each $L=\left\{\left\{H_{\alpha \beta}: \beta \in B_{\alpha}\right\}: \alpha \in A\right\} \in \mathscr{G}$ we define $\bar{L}=\bigvee_{\alpha \in A} \bigwedge_{\beta \in B_{x}} \bar{H}_{\alpha \beta} \subset G$. define:

Definition 2.1. Let $L=\left\{L_{\alpha}\right\}=\left\{\left\{H_{\alpha \beta}\right\}\right\}, M=\left\{M_{\gamma}\right\}=\left\{\left\{H_{\gamma \delta}\right\}\right\} \in \mathscr{G}$. We

(i) $L+M=\left\{\left\{H_{\alpha \beta}+H_{\gamma \delta}:(\beta, \delta) \in B_{\alpha} \times \Delta_{\gamma}\right\}:(\alpha, \gamma) \in A \times \Gamma\right\}$;

(ii) $-L=\left\{\left\{-H_{\alpha f(\alpha)}: \alpha \in A\right\}: f \in \chi_{\alpha \in A} B_{\alpha}\right\}$;

(iii) $L-M=L+(-M),-L+M=(-L)+M$;

(iv) $L \vee M=L \cup M$;

(v) $L \wedge M=\left\{L_{\alpha} \cup M_{\gamma}:(\alpha, \gamma) \in A \times \Gamma\right\}$.

In (ii) the Cartesian product $\chi_{\alpha \in A} B_{\alpha}$ is the set of choice functions $f$ with domain $A$ such that $f(\alpha) \in B_{\alpha}(\alpha \in A)$.

The definitions above make $\mathscr{G}$ an associative semigroup under addition which is also closed under the other operations. Furthermore, given any $\mathscr{L}$ completion of $G$ we have a natural map of $\mathscr{G}$ into the $\mathscr{L}$-completion which takes each $\{\{(M)\}\}(M \in \mathscr{D})$ to $\vee M$ and reduces the operations in $\mathscr{G}$ to ordinary lattice operations in the $\mathscr{L}$-completion. We proceed to exploit this.

Definitions 2.2. Let $H=\left(\varepsilon_{1} M_{1}, \cdots \varepsilon_{n} M_{n}\right)$. A negative part of $H$ is any element $\left(\varepsilon_{1} M_{1}^{*}, \cdots, \varepsilon_{n} M_{n}^{*}\right)$ of $\mathscr{H}$ such that $M_{i}^{*}$ is a singleton subset of $M_{i}$ if $\varepsilon_{i}=1$ and $M_{i}^{*}=M_{i}$ if $\varepsilon_{i}=-1$. When $\varepsilon_{i}=1$ we say the negative part of $H$ fixes the singleton $M_{i}^{*}$ in $M_{i}$. A positive part of $H$ is defined dually by fixing a singleton in each $M_{i}$ such that $\varepsilon_{i}=-1$.

For $L=\left\{\left\{H_{\alpha \beta}\right\}\right\} \in \mathscr{G}$, a negative (positive) subsystem of $L$ is an element $\left\{\left\{N_{\alpha \beta}\right\}\right\}\left(\left\{\left\{P_{\alpha \beta}\right\}\right\}\right)$ of $\mathscr{G}$ such that for each $\alpha, \beta, N_{\alpha \beta}$ is a negative part of $H_{\alpha \beta}\left(P_{\alpha \beta}\right.$ is a positive part of $H_{\alpha \beta}$ ).

Next we define three subsets (in fact subsemigroups) of $\mathscr{G}$.

First, $\mathscr{K}^{-}$is the set of all elements $L \in \mathscr{G}$ such that for any negative subsystem $N=\left\{\left\{N_{\alpha \beta}\right\}\right\}$ of $L$,

$$
\inf \vec{N}^{+}=\inf \left(\bigvee_{\alpha} \bigwedge_{\beta} \bar{N}_{\alpha \beta}\right)^{+}=\inf \left(\bigvee_{\alpha} \bigwedge_{\beta} \bar{N}_{\alpha \beta}^{+}\right)=0
$$

(We use 'inf' here to denote the infimum in $G$ so as to avoid confusion with our subset calculus.)

Second, $\mathscr{K}^{+}$is the set of $L \in \mathscr{G}$ such that inf $\bar{P}^{-}=0$ for every positive system $P$ of $L$.

Finally, $\mathscr{K}=\mathscr{K}^{+} \cap \mathscr{K}^{-}$.

Before starting on the proof of the next lemmas we make three useful observations: 
(A) If $V=p\left(U_{1}, \cdots, U_{n}\right)$ is a finite combination of subsets of $G$ using addition, supremum and infimum, the set of lower bounds and hence the infimum if it exists, of $V$ are unchanged by replacing any set $U_{i}$ by $\downarrow U_{i}$, the set of all finite infima of elements of $U_{i}$.

(B) Let $U_{\alpha \beta}\left(\beta \in B_{\alpha}, \alpha \in A\right)$ be finitely many non-empty subsets of $G$ and let $u_{\alpha \beta} \in U_{\alpha \beta}$ for all $\alpha, \beta$. Since $\bigvee_{\alpha \in A} \bigwedge_{\beta \in B_{z}} u_{\alpha \beta}=\bigwedge_{f \in \times B_{x}} \bigvee_{\alpha} u_{\alpha f,(\alpha)}$, it follows that $\bigvee_{\alpha} \wedge_{\beta} U_{\alpha \beta} \subset \bigwedge_{f} \bigvee_{\alpha} U_{\alpha f(\alpha)}$. If $u_{\alpha f(\alpha)} \in U_{\alpha f(\alpha)}$ for each $f$ and $\alpha$ we can find $v_{\alpha \beta} \in \downarrow U_{\alpha \beta}\left(\beta \in B_{\alpha}, \alpha \in A\right)$ such that $v_{\alpha \beta} \leqq u_{\alpha f(\alpha)}(f(\alpha)=\beta)$. Then $\bigvee_{a} \wedge_{\beta} v_{\alpha \beta}$ $=\bigwedge_{f} \bigvee_{\alpha} v_{\alpha f(\alpha)} \leqq \bigwedge_{f} \bigvee_{\alpha} u_{\alpha f(\alpha)}$. Since $\left.\inf \left(\bigvee_{\alpha} \wedge_{\beta} U_{\alpha \beta}\right)=\inf \left(\bigvee_{\alpha} \wedge_{\beta} \downarrow U_{\alpha \beta}\right)\right)$ (if either exists) it follows that inf $\bigvee_{a} \wedge_{\beta} U_{\alpha \beta}=\inf \wedge_{f} \vee_{\alpha} U_{\alpha f(\alpha)}$, if either infimum exists.

The dual result, inf $\wedge_{\alpha} \bigvee_{\beta} U_{\alpha \beta}=\inf \bigvee_{f} \wedge_{\alpha} U_{\alpha f(x)}$ is proved similarly.

(C) From infinite distributivity of the lattice operations in $G$ we deduce that $\inf \left(U_{1} \vee U_{2}\right)=\left(\inf U_{1}\right) \vee\left(\inf U_{2}\right)$ for any subsets $U_{1}, U_{2}$ of $G$ which have infima. A sssociativity also gives $\inf \left(U_{1} \wedge U_{2}\right)=\left(\inf U_{1}\right) \wedge\left(\inf U_{2}\right)$.

For Lemmas 2.3, 2.4, 2.5 we assume $L=\left\{\left\{H_{\alpha \beta}: \beta \in B_{\alpha}\right\}: \alpha \in A\right\}$ and $K=\left\{\left\{H_{\gamma \delta}: \delta \in \Delta_{\gamma}\right\}: \gamma \in \Gamma\right\}$ are elements of $\mathscr{G}$.

Lemma 2.3. (i) If $K, L \in \mathscr{K}^{-}$then $K+L \in \mathscr{K}^{-}$.

(ii) If $K, L \in \mathscr{K}^{+}$then $K+L \in \mathscr{K}^{+}$.

(iii) If $K, L \in \mathscr{K}$ then $K+L \in \mathscr{K}$.

ProOF. (i) Let $Q$ be a negative system of $K+L$, then

$Q=\left\{\left\{R_{\alpha \gamma \beta \delta}+S_{\alpha \gamma \beta \delta}:(\beta, \delta) \in B_{\alpha} \times \Delta_{\gamma}\right\}:(\alpha, \gamma) \in A \times \Gamma\right\}$ where each $R_{\alpha \gamma \beta \delta}$, $S_{\alpha \gamma \beta \delta}$ are negative parts of $H_{\alpha \beta}, H_{\gamma \delta}$. This gives

$$
\bar{Q}^{+}=\bigvee_{\alpha, \gamma} \bigwedge_{\beta, \delta}\left(\bar{R}_{\alpha \gamma \beta \delta}+\bar{S}_{\alpha \gamma \beta \delta}\right)^{+} .
$$

By (C) to show inf $\bar{Q}^{+}=0$ it is sufficient to show that for each $\alpha, \gamma$

$$
\inf \bigwedge_{\beta, \delta}\left(\bar{R}_{\alpha \gamma \beta \delta}+S_{\alpha \gamma \beta \delta}\right)^{+}=0 .
$$

Let $r_{\alpha \gamma \beta \delta} \in \bar{R}_{\alpha \gamma \beta \delta}, s_{\alpha \gamma \beta \delta} \in S_{\alpha \gamma \beta \delta}$, then

$$
\begin{aligned}
\bigwedge_{\beta, \delta}\left(r_{\alpha \gamma \beta \delta}+s_{\alpha \gamma \beta \delta}\right)^{+} & \leqq \bigwedge_{\beta, \delta}\left(r_{\alpha \gamma \beta \delta}^{+}+s_{\alpha \gamma \beta \delta}^{+}\right) \\
& \leqq \bigwedge_{\beta}\left[\bigvee_{\delta} r_{\alpha \gamma \beta \delta}^{+}+\bigwedge_{\delta} s_{\alpha \gamma \beta \delta}^{+}\right] \leqq \bigwedge_{\beta} \bigvee_{\delta} r_{\alpha \gamma \beta \delta}^{+}+\bigvee_{\beta} \bigwedge_{\delta} s_{\alpha \gamma \beta \delta}^{+} .
\end{aligned}
$$

Hence

$$
\begin{aligned}
\inf \left(\bigwedge_{\beta, \delta}\left(\tilde{R}_{\alpha \gamma \beta \delta}+\bar{S}_{\alpha \gamma \beta \delta}\right)^{+}\right) & \leqq \inf \left[\bigwedge_{\beta} \underset{\delta}{\bigvee} \bar{R}_{\alpha \gamma \beta \delta}^{+}+\bigvee_{\beta} \bigwedge_{\delta} S_{\alpha \gamma \beta \delta}^{+}\right] \\
& =\inf \left(\bigwedge_{\beta} \underset{\delta}{\bigvee} \bar{R}_{\alpha \gamma \beta \delta}^{+}\right)+\inf \left(\underset{\beta}{\vee} \bigwedge_{\delta} S_{\alpha \gamma \beta \delta}^{+}\right) .
\end{aligned}
$$


By (C),

$$
\inf \left(\bigvee_{\beta} \bigwedge_{\delta} S_{\alpha \gamma \beta \delta}^{+}\right)=\bigvee_{\beta}\left[\inf \left(\bigwedge_{\delta} S_{\alpha \gamma \beta \delta}^{+}\right)\right]=0
$$

since for each $\alpha \gamma \beta, S_{\alpha \gamma \beta \delta}$ is a negative part of $H_{\gamma \delta}$ and $L \in \mathscr{K}^{-}$.

By (B) and (C),

$$
\begin{aligned}
\inf \left(\bigwedge_{\beta} \bigvee_{\delta} \bar{R}_{\alpha \gamma \beta \delta}^{+}\right) & =\inf \left(\bigvee\left\{\bigwedge_{\beta} \bar{R}_{\alpha \gamma \beta f(\beta)}^{+}: f \in \Delta_{\gamma}^{B_{\gamma}}\right\}\right) . \\
& =\bigvee_{f}\left[\inf \left(\bigwedge_{\beta} \bar{R}_{\alpha \gamma \beta f(\beta)}^{+}\right)\right]=0,
\end{aligned}
$$

since for each $\alpha, \gamma, f, R_{\alpha \gamma \beta f(\beta)}$ is a negative part of $H_{\alpha \beta}$ and $K \in \mathscr{K}^{-}$. We conclude that inf $\wedge_{\beta, \delta}\left(\bar{R}_{\alpha \gamma \beta \delta}+S_{\alpha \gamma \beta \delta}\right)^{+}=0$ for each $\alpha, \gamma$ and hence $K+L \in \mathscr{K}^{-}$.

(ii) Let $Q$ be positive subsystem of $K+L$, then $Q=\left\{\left\{R_{\alpha \gamma \beta \delta}+S_{\alpha \gamma \beta \delta}\right\}\right\}$ with $R_{\alpha \gamma \beta \delta}, S_{\alpha \gamma \beta \delta}$ positive parts of $H_{\alpha \beta}, H_{\gamma \delta}$. Then, by (B),

$$
\begin{aligned}
\inf \bar{Q}^{-} & =\inf \bigwedge_{\alpha, \gamma} \underset{\beta, \delta}{\bigvee}\left(\bar{R}_{\alpha \gamma \beta \delta}+S_{\alpha \gamma \beta \delta}\right)^{-} \\
& =\inf \vee\left\{\bigwedge_{\alpha, \gamma}\left(\bar{R}_{\alpha \gamma q r}+S_{\alpha \gamma q r}\right)^{-}:(q, r) \in \underset{(\alpha, \gamma) \in A \times \Gamma}{\times} B_{\alpha} \times \underset{(\alpha, \gamma) \in A \times \Gamma}{\times} \Delta_{\gamma}\right\} .
\end{aligned}
$$

(for each $\alpha, \gamma, q, r$, we have written $R_{\alpha \gamma q r}$ for $R_{\alpha \gamma q(\alpha, \gamma) r(\alpha, \gamma)}$ and similarly for $S_{\alpha, q r}$ ). We require inf $\bar{Q}^{-}=0$ and, by (C), it is sufficient to show that inf $\Lambda_{\alpha, \gamma}\left(\bar{R}_{\alpha \gamma q r}+S_{\alpha \gamma q r}\right)^{-}=0$ for each $q, r$.

Now, as in (i), we have

$$
\begin{aligned}
\inf \bigwedge_{\alpha, \gamma}\left(\bar{R}_{\alpha \gamma q r}+S_{\alpha \gamma q r}\right)^{-} & \leqq \inf \left[\bigwedge_{\alpha, \gamma}\left(\bar{S}_{\alpha \gamma q r}^{-}+\bar{R}_{\alpha \gamma q r}^{-}\right)\right] \\
& \leqq \inf \left(\bigvee_{\alpha} \bigwedge_{\gamma} \bar{S}_{\alpha \gamma q r}^{-}\right)+\inf \left(\bigwedge_{\alpha} \bigvee_{\gamma} \bar{R}_{\alpha \gamma q r}^{-}\right) .
\end{aligned}
$$

For fixed $\alpha, S_{\alpha \gamma q r}$ is a positive part of $H_{\gamma r(\alpha, \gamma)}$ and $r(\alpha,) \in \chi_{\gamma \in \Gamma} \Delta_{\gamma}$. Because $L \in \mathscr{K}^{+},(B)$ gives

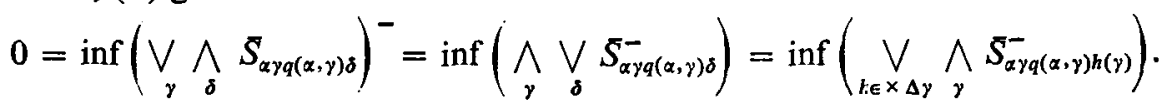

Hence, $\left.\inf \left(\bigvee_{\alpha} \wedge_{\gamma} S_{\alpha q_{q} r}^{-}\right)=\bigvee_{\alpha} \inf \wedge_{\gamma} \bar{S}_{\alpha \gamma_{q r}}^{-}\right)=0$.

Similarly,

$$
\inf \left(\bigwedge_{\alpha} \bigvee_{y} \bar{R}_{\alpha q q r}^{-}\right)=\inf \bigvee_{f \in \Gamma^{A}} \bigwedge_{\alpha} \bar{R}_{\alpha f(\alpha) q r}^{-}=\bigvee_{f}\left[\inf \left(\bigwedge_{\alpha} \bar{R}_{\alpha f(\alpha) q r}^{-}\right)\right]=0
$$

because, $q(, f()) \in X_{\alpha \in A} B_{\alpha}, R_{\alpha f(\alpha) q(\alpha, f(\alpha)) r(\alpha, f(\alpha))}$ is a positive part of $H_{\alpha q(\alpha f(a))}$ and $K \in \mathscr{K}^{+}$.

Thus we have $\inf \bar{Q}^{-}=0$ and $K+L \in \mathscr{K}^{+}$as required.

(iii) This part follows from (i) and (ii). 
LEMMA 2.4.

(i) If $K \in \mathscr{K}^{-}$then $L+K-L \in \mathscr{K}^{-}$and $-L+K+L \in \mathscr{K}^{-}$.

(ii) If $K \in \mathscr{K}^{+}$then $L+K-L \in \mathscr{K}^{+}$and $-L+K+L \in \mathscr{K}^{+}$.

(iii) If $K \in \mathscr{K}$ then $L+K-L \in \mathscr{K}$ and $-L+K+L \in \mathscr{K}$.

In particular $L-L \in \mathscr{K}$ and $-L+L \in \mathscr{K}(L \in \mathscr{G})$.

Proof. (i) Let $Q$ be a negative subsystem of $L+K-L$, then

$$
\begin{gathered}
Q=\left\{\left\{R_{\alpha \gamma \delta \beta \delta \eta}+N_{\alpha \gamma \delta \beta \delta \eta}-S_{\alpha \gamma \delta \beta \delta \eta}:(\beta, \delta, \eta) \in B_{\alpha} \times \Delta_{\gamma} \times A\right\}:\right. \\
(\alpha, \gamma, f) \in A x \Gamma \times \underset{\alpha \in A}{\times} B_{\alpha} ;
\end{gathered}
$$

with, for each choice of subscripts $R_{\alpha \gamma \delta \beta \delta \eta}$ a negative part of $H_{\alpha \beta}, N_{\alpha \gamma f \beta \delta \eta}$ a negative part of $H_{\gamma \delta}$ and $S_{\alpha \gamma \delta \beta \delta \eta}$ a positive part of $H_{\eta f(\eta)}$.

Suppose that

$$
H_{\alpha \beta}=\left(\varepsilon(\alpha, \beta)_{1}, M(\alpha, \beta)_{1}, \cdots, \varepsilon(\alpha, \beta)_{n(\alpha, \beta)} M(\alpha, \beta)_{n(\alpha, \beta)}\right) .
$$

We define finite subsets $M^{\prime}(\alpha, \beta)_{i}$ of $M(\alpha, \beta)_{i}$ as follows. If $\varepsilon(\alpha, \beta)_{i}=1, M^{\prime}(\alpha, \beta)_{i}$ is the union of the set of singletons fixed in $M(\alpha, \beta)_{i}$ by the $R_{\alpha \gamma \delta \beta \delta \eta}$ as $\gamma, f, \delta, \eta$ vary. If $\varepsilon(\alpha, \beta)_{i}=-1, M^{\prime}(\alpha, \beta)_{i}$ is the union of the set of singletons fixed in $M(\alpha, \beta)_{i}$ by the $S_{\alpha^{\prime} \gamma \delta \beta^{\prime} \delta \alpha}$ as $\alpha^{\prime}, \gamma, \beta^{\prime}, \delta$ and $f$ vary such that $f(\alpha)=\beta$.

Define $R_{\alpha \gamma f \beta \delta \eta}^{\prime}$ by replacing, in $R_{\alpha \gamma \delta \beta \delta \eta}$, each $M(\alpha, \beta)_{i}$ for which $\varepsilon(\alpha, \beta)_{i}=-1$ by $M^{\prime}(\alpha, \beta)_{i}$. Similarly define $S_{\alpha \gamma \delta \beta \delta \eta}^{\prime}$ by replacing, in $S_{\alpha \gamma f \beta \delta \eta}$, each $M(\eta, f(\eta))_{j}$ for which $\varepsilon(\eta, f(\eta))_{j}=1$ by $M^{\prime}(\eta, f(\eta))_{j}$. This leads to

$$
\bar{Q} \supset \underset{\alpha \gamma \delta}{\bigvee} \bigwedge_{\beta \delta \eta}\left(\bar{R}_{\alpha \gamma \delta \beta \delta \eta}^{\prime}+\bar{N}_{\alpha \gamma \delta \beta \delta \eta}-S_{\alpha \gamma \delta \beta \delta \eta}^{\prime}\right)=\bar{Q}_{1} \text { say. }
$$

It is now sufficient to show that $\inf \bar{Q}_{1}^{+}=0$. We apply (A) to $Q_{1}$ and replace, in each $R_{\alpha \gamma \delta \beta \delta \eta}^{\prime}$, those $\varepsilon(\alpha, \beta)_{i} M^{\prime}(\alpha, \beta)_{i}$ for which $\varepsilon(\alpha, \beta)_{i}=-1$ by $\downarrow\left(\varepsilon(\alpha, \beta)_{i} M^{\prime}(\alpha, \beta)_{i}\right)$. Since $M^{\prime}(\alpha, \beta)_{i}$ is finite we then retain only the term $\wedge\left(\varepsilon(\alpha, \beta)_{i} M^{\prime}(\alpha, \beta)_{i}\right)=$ $=\varepsilon(\alpha, \beta)_{i} \bigvee M^{\prime}(\alpha, \beta)_{i}$. This leads to $R_{\alpha \gamma \delta \beta \delta \eta}^{\prime \prime}$ such that $\bar{R}_{\alpha \gamma \delta \beta \delta \eta}^{\prime \prime}$ is a singleton, say $r_{\alpha \gamma \delta \beta S_{\eta}}$. Taking account of the sign preceding $S_{\alpha \gamma \delta \beta \delta \eta}^{\prime}$ we can form $S_{\alpha \gamma f \beta \delta \eta}^{\prime \prime}$ by replacing each $M(\eta, f(\eta))_{j}$ with $\varepsilon(\eta, f(\eta))_{j}=1$ by $\left\{\bigvee M^{\prime}(\eta, f(\eta))_{j}\right\}$. Then

$$
\inf \bar{Q}_{1}^{+}=\inf \underset{\alpha \gamma \delta}{\bigvee} \bigwedge_{\beta \delta \eta}\left(\bar{R}_{\alpha \gamma f \beta \delta \eta}^{\prime \prime}+\bar{N}_{\alpha \gamma f \beta \delta \eta}-S_{\alpha \gamma f \beta \delta \eta}^{\prime \prime}\right)^{+}
$$

Now in $R_{\alpha \gamma \delta \beta \delta \eta}^{\prime \prime}$ each singleton subset of $M(\alpha, \beta)_{i}$ with $\varepsilon(\alpha, \beta)_{i}=1$ is contained in $M^{\prime}(\alpha, \beta)_{i}$. It follows that

$$
\begin{aligned}
r_{\alpha \gamma f \beta \delta \eta} \leqq & \varepsilon(\alpha, \beta)_{1} \bigvee M^{\prime}(\alpha, \beta)_{1}+\cdots+\varepsilon(\alpha, \beta)_{n(\alpha \beta)} \vee M^{\prime}(\alpha, \beta)_{n(\alpha, \beta)} \\
& =g_{\alpha \beta} \text { say. }
\end{aligned}
$$

Similarly, if $S_{\alpha \gamma f \beta \delta \eta}^{\prime \prime}=\left\{s_{\alpha \gamma \delta \beta \delta \eta}\right\}$, we have $-s_{\alpha \gamma f \beta \delta \eta} \leqq-g_{\eta f(\eta)}$ where $g_{\eta \delta(\eta)}$ is defined as above for $g_{\alpha \beta}$. 
This gives

$$
\begin{aligned}
\inf \bar{Q}_{1}^{+} & \left.\leqq \inf \bigvee_{\alpha \gamma f} \bigwedge_{\beta \delta \eta}\left(g_{\alpha \beta}+\bar{N}_{\alpha \gamma f \beta \delta \eta}-g_{\eta f(\eta)}\right)^{+}\right] \\
& \left.\leqq \inf \bigvee_{\alpha \gamma f} \bigwedge_{\delta}\left(g_{\alpha f(\alpha)}+\bar{N}_{\alpha \gamma f f(\alpha) \delta \alpha}-g_{\alpha f(\alpha)}\right)^{+}\right] \\
& \left.=\bigvee_{\alpha \gamma f} \inf \bigwedge_{\delta}\left(g_{\alpha f(\alpha)}+\bar{N}_{\alpha \gamma f f(\alpha) \delta \alpha}^{+}-g_{\alpha f(\alpha)}\right)\right] \\
& \left.=\bigvee_{\alpha \gamma f} g_{\alpha f(\alpha)}+\inf \bigwedge_{\delta} \bar{N}_{\alpha \gamma f f(\alpha) \delta \alpha}^{+}-g_{\alpha f(\alpha)}\right] \\
& =\bigvee_{\alpha \gamma f}\left[g_{\alpha f(\alpha)}+0-g_{\alpha f(\alpha)}\right]=0 .
\end{aligned}
$$

(The equality inf $\wedge_{\delta} \bar{N}_{\alpha \gamma f f(\alpha) \delta \alpha}^{+}=0$ follows because $K \in \mathscr{K}^{-}$and for each $\alpha, f, N_{\alpha \gamma f f(\alpha) \delta \alpha}$ is a negative part of $H_{\gamma \delta}$.)

This shows that $L+K-L \in \mathscr{K}^{-}$; the proof that $-L+K+L \in \mathscr{K}^{-}$is entirely similar.

(ii) This time let $Q$ be a positive sybsystem of $L+K-L$ then

$$
Q=\left\{\left\{R_{\alpha \gamma \delta \beta \delta \eta}+P_{\alpha \gamma \delta \beta \delta \eta}-S_{\alpha \gamma \delta \beta \delta \eta}\right\}\right\}
$$

with each $R_{\alpha \gamma \delta \beta \delta \eta}$ a positive part of $H_{\alpha \beta}, P_{\alpha \gamma \delta \beta \delta \eta}$ a positive part of $H_{\gamma \delta \delta}$ and $S_{\alpha \gamma f \eta \beta \delta}$ a negative part of $H_{\eta f(\eta)}$. We require

$$
0=\inf \bar{Q}^{-}=\inf \left[\bigwedge_{\alpha \gamma f} \underset{\gamma \delta \eta}{V}\left(\bar{S}_{\alpha \gamma f \beta \delta \eta}-\bar{P}_{\alpha \gamma \delta \beta \delta \eta}-\bar{R}_{\alpha \gamma \delta \beta \delta \eta}\right)^{+}\right]
$$

Arguing in much the same way as in part (i) we find elements $g_{\alpha \beta} \in G\left(\beta \in B_{\alpha}, \alpha \in A\right)$ such that

$$
\begin{aligned}
\inf \bar{Q}^{-} & \leqq \inf \left[\bigwedge_{\alpha \gamma \delta} \bigvee_{\beta \delta \eta}\left(g_{\eta f(\eta)}+\bar{P}_{\alpha \gamma \delta \beta \delta \eta}^{-}-g_{\alpha \beta}\right)^{+}\right] \\
& \leqq \inf \left[\bigwedge_{\alpha \gamma f} \bigvee_{\beta \delta \eta}\left(\left(\bigvee_{\eta} g_{\eta f(\eta)}\right)+\bar{P}_{\alpha \gamma \delta \beta \delta \eta}^{-}-\left(\bigwedge_{\beta} g_{\alpha \beta}\right)\right)^{+}\right]
\end{aligned}
$$

Write $h_{f}=\bigvee_{\eta} g_{\eta f(\eta)}$ and $g_{\alpha}=\bigwedge_{\beta} g_{\alpha \beta}$.

Then,

$$
\begin{aligned}
\inf Q^{-} & \leqq \bigwedge_{\alpha f} \inf \left[\bigwedge_{\gamma} \bigvee_{\beta \delta \eta}\left(h_{f}+\bar{P}_{\alpha \gamma f \beta \delta \eta}^{-}-g_{\alpha}\right)^{+}\right] \\
& =\bigwedge_{\alpha f}\left(h_{f}+\inf \left[\bigwedge_{\gamma} \bigvee_{\beta \eta} \underset{\delta}{\bigvee} \bar{P}_{\alpha \gamma \delta \beta \delta \eta}^{-}\right]-g_{\alpha}\right)^{+}
\end{aligned}
$$

Now, by (B) and (C),

$$
\begin{aligned}
\inf & {\left[\bigwedge_{\gamma} \underset{\beta, \eta}{\bigvee} \bigvee_{\delta} \bar{P}_{\alpha \gamma f \beta \delta \eta}^{-}\right]=\inf \underset{(q, r) \in\left(B_{\alpha} \times A\right)}{\bigvee} \bigwedge_{\gamma} \bigvee_{\delta} \bar{P}_{\alpha \gamma f q(\gamma) \delta r(\gamma)}^{-} } \\
& =\bigvee_{q, r} \inf \left[\bigwedge_{\gamma} \underset{\delta}{\bigvee} \bar{P}_{\alpha \gamma f q(\gamma) \delta r(\gamma)}^{-}\right]=\bigvee_{q, r} \inf \left[\left(\bigvee_{\gamma} \bigwedge_{\delta} \bar{P}_{\alpha \gamma f q(\gamma) \delta r(\gamma)}\right)^{-}\right] .
\end{aligned}
$$


This last expression is zero since each $P_{\alpha \gamma f q(\gamma) \delta r(\gamma)}$ is a positive part of $H_{\gamma \delta}$ and $K \in \mathscr{K}^{+}$. Hence

$$
\inf \bar{Q}^{-} \leqq \bigwedge_{\alpha, f}\left(h_{f}-g_{\alpha}\right)^{+}=\left[\bigwedge_{f} \bigvee_{\eta} g_{\eta f(\eta)}-\bigvee_{\alpha} \bigwedge_{\beta} g_{\alpha \beta}\right]^{+}=0 .
$$

Thus $L+K-L$, and similarly $-L+K+L$, is in $\mathscr{K}+$.

(iii) This part is immediate from (i) and (ii).

The arguments above apply if $K=\varnothing$. This gives the final statement of this Lemma.

LEMMA 2.5 .

(i) If $K \in \mathscr{K}^{+}$and $L+K \in \mathscr{K}^{-}$or $K+L \in \mathscr{K}^{-}$, then $L \in \mathscr{K}^{-}$.

(ii) If $K \in \mathscr{K}^{-}$and $L+K \in \mathscr{K}^{+}$or $K+L \in \mathscr{K}^{+}$, then $L \in \mathscr{K}^{+}$.

Proof. Let $R=\left\{\left\{R_{\alpha \beta}\right\}\right\}$ be a negative subsystem of $L$ and $N=\left\{\left\{N_{\gamma \delta}\right\}\right\}$ a negative subsystem of $K$; then $R+N$ is a negative subsystem of $L+K \in \mathscr{K}^{-}$. We verify first that $\inf (\bar{R}+\bar{N})^{+}=0$.

Since $L+K \in \mathscr{K}^{-}$,

$$
0=\inf (\overline{R+N})^{+}=\inf \left[\bigvee_{\alpha \gamma} \bigwedge_{\beta \gamma \delta}\left(\bar{R}_{\alpha \beta}+\bar{N}_{\gamma \delta}\right)^{+}\right] .
$$

Let $r_{\alpha \gamma \beta \delta} \in \bar{R}_{\alpha \beta}, n_{\alpha \gamma \beta \delta} \in \bar{N}_{\gamma \delta}$ and put $r_{\alpha \beta}=\bigwedge_{\gamma \in \Gamma} \bigwedge_{\delta \in \Delta \gamma} r_{\alpha \gamma \beta \delta} \in \downarrow \bar{R}_{\alpha \beta}, n_{y \delta}=$ $\bigwedge_{\alpha} \wedge_{\beta \in B_{z}} n_{a \gamma \beta \delta} \in \downarrow \bar{N}_{\gamma \delta}$. Then $\bigvee_{\alpha \gamma} \bigwedge_{\beta \delta}\left(r_{\alpha \beta}+n_{\gamma \delta}\right)^{+}=\left(\bigvee_{\alpha} \wedge_{\beta} r_{\alpha \beta}+\bigvee_{\gamma} \bigwedge_{\delta} n_{\gamma \delta}\right)^{+}$.

By (A),

$0 \leqq \inf (\bar{R}+\bar{N})^{+} \leqq\left(\bigvee_{\alpha} \bigwedge_{\beta} r_{\alpha \beta}+\bigvee_{\gamma} \bigwedge_{\delta} n_{\gamma \delta}\right)^{+} \leqq \bigvee_{\alpha, \gamma} \bigwedge_{\beta, \delta}\left(r_{\alpha \gamma \beta \delta}+n_{\alpha \gamma \beta \delta}\right)^{+} \epsilon(\overline{R+N})^{+}$.

Hence $\inf \left((\bar{R}+\bar{N})^{+}=0\right.$.

Now suppose $g \geqq 0$ and $g$ is a lower bound for $\bar{R}^{+}$. If $u \in \bar{R}, v \in \bar{N}$, then $(u+v)^{+} \geqq\left(u^{+}-u^{-}-v^{-}\right)^{+}=u^{+}-u^{+} \wedge v^{-}=\left(u^{+}-v^{-}\right)^{+} \geqq\left(g-v^{-}\right)^{+}$ $=g-g \wedge v^{-}$. It follows that $\inf \left\{g-g \wedge v^{-}: v \in \bar{N}\right\}=0$, and hence $g=\sup \left\{g \wedge v^{-}: v \in \bar{N}\right\}$, for every negative subsystem $N$ of $K$.

Fix $\gamma_{0} \in \Gamma$ and $\delta_{0} \in \Delta_{\gamma_{0}}$ and suppose

$$
H_{\gamma_{0} \delta_{o}}=\left(\varepsilon_{1} M_{1}, \cdots, \varepsilon_{k} M_{k}, \varepsilon_{k+1} M_{k+1}, \cdots, \varepsilon_{j} M_{j}, M_{j+1}, \cdots, M_{n}\right) \text { where } M_{1}, \cdots, M_{k}
$$

are singletons (possibly there are no such terms) and $g \perp M_{r}(r=j+1, \cdots, n)$ (again there may be no such terms).

We shall modify $K$ to $K^{*}=\left\{\left\{H_{y d}^{*}\right\}\right\}$ and replace $g$ by $g^{*}$ so that: (i) $g^{*}>0$ if $g>0$; (ii) $K^{*} \in \mathscr{K}^{+}$; (iii) $g^{*}=\sup \left\{g^{*} \wedge v^{-}: v \in \bar{N}^{*}\right\}$ for every negative subsystem $N^{*}$ of $K^{*}$. There are four cases to consider.

CASE 1. $\varepsilon_{j}=1, g \perp M_{j}$; take $K^{*}=K$ and $g^{*}=g$.

CASE 2. $\varepsilon_{j}=1$ and there exists $m \in M_{j}$ such that $g \wedge m \neq 0$. Choose one such $m$, put $g^{*}=g \wedge m, M^{*}=M_{j} \sim\{m\}, H_{\gamma \delta}^{*}=H_{\gamma \delta}$ if $(\gamma, \delta) \neq\left(\gamma_{0}, \delta_{0}\right)$, and 
$H_{\gamma_{0} \delta_{0}}^{*}=\left(\{m\}, \varepsilon_{1}\left(-m+M_{1}+m\right), \cdots, \varepsilon_{j-1}\left(-m+M_{j-1}+m\right), M^{*}, M_{j+1}, \cdots, M_{n}\right)$. This defines $K^{*}=\left\{\left\{H_{y \delta}^{*}\right\}\right\}$. Observe that for any $x, u, a, b$ in a lattice group such that $x \geqq 0$ and $x \perp a$ and $x \perp b$,

$$
x \wedge(u+a)^{-}=x \wedge u^{-}=x \wedge(u+b)^{-} .
$$

(We have $x \wedge(u+a)^{-} \leqq x \wedge\left(a^{-}+u^{-}\right) \leqq x \wedge a^{-}+x \wedge u^{-}=x \wedge u^{-}$ $\left.=x \wedge(u+a-a)^{-} \leqq x \wedge\left((-a)^{-}+(u+a)^{-}\right)=x \wedge(u+\mathrm{a})^{-}.\right)$

Let $P_{\gamma_{0} \delta_{0}}^{*}$ be a positive part of $H_{\gamma_{0} \delta_{0}}^{*}$. By fixing the same singletons in the $M_{r}$ with $\varepsilon_{r}=-1$ we have a corresponding positive part $P_{\gamma_{0} \delta_{0}}$ of $H_{\gamma_{0} \delta_{0}}$. Let $p^{*} \in \bar{P}_{\gamma_{0} \delta_{0}}^{*}$ then $p^{*}=u+m+m^{\prime}+v$ with $u \in \varepsilon_{1} M_{1}+\cdots+\varepsilon_{j-1} M_{j-1}$ (the conjugations cancel), $m^{\prime} \in M^{*}$ and $v \in M_{j+1}+\cdots+M_{n}$. Now, $p^{*}=(u+m+v) \vee\left(u+m^{\prime}+v\right)$ because $m \wedge m^{\prime}=0$. Since every element of $\bar{P}_{\gamma_{0} \delta_{0}}$ is either of the form $u+m+v$ or $u+m^{\prime}+v$ with $m^{\prime} \in M^{*}$, every $p^{-} \in \bar{P}_{\gamma_{0} \delta_{0}}^{-}$exceeds some $p^{*-} \in \bar{P}_{\gamma_{0} \delta_{0}}^{*-}$. Since $H_{\gamma \delta}^{*}=H_{\gamma \delta}$ if $(\gamma, \delta) \neq\left(\gamma_{0}, \delta_{0}\right)$ we conclude that if $P^{*}$ is a positive subsystem of $K^{*}$ and $P$ the corresponding positive subsystem of $K$,

$$
\inf \bar{P}^{*-} \leqq \inf \bar{P}^{-}=0 \text {, }
$$

and $K^{*} \in \mathscr{K}^{+}$.

Let $N^{*}=\left\{\left\{N_{\gamma \delta}^{*}\right\}\right\}$ be a negative subsystem of $K$. Corresponding to $N_{\gamma_{0} \delta_{0}}^{*}$ we have a negative part $N_{\gamma_{0} \delta_{0}}$ of $H_{\gamma_{0} \delta_{0}}$ obtained by fixing $m$ in $M_{j}$ and the same singletons in the other $M_{r}$ with $\varepsilon_{r}=1$. If $n \in \bar{N}_{\gamma_{0} \delta_{0}}$ we have $n=u+m+v$ and $n^{*}=u+m+m^{\prime}+v \in \bar{N}_{\gamma_{0} \delta_{0}}^{*}$. Since $g^{*} \perp m^{\prime}+v$ and $g^{*} \perp v, g^{*} \wedge n^{-}=g^{*} \wedge u^{-}$ $=g^{*} \wedge n^{*-}$. Taking $N_{\gamma \delta}=N_{\gamma \delta}^{*}$ if $(\gamma, \delta) \neq\left(\gamma_{0}, \delta_{0}\right)$ we have $g^{*} \wedge \bar{N}_{\gamma \delta}=g^{*} \wedge \bar{N}_{\gamma \delta}^{*-}$ for all $\gamma, \delta$ and hence

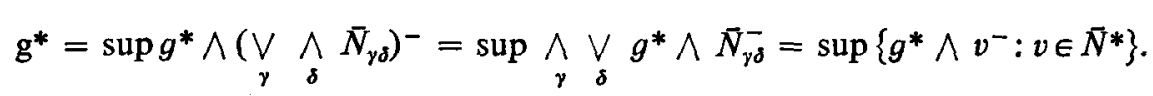

This concludes Case 2.

CASE 3. $\varepsilon_{j}=-1, g \perp M_{j}$. Take $g^{*}=g, H_{\gamma \delta}^{*}=H_{\gamma \delta}\left((\gamma, \delta) \neq\left(\gamma_{0}, \delta_{0}\right)\right)$, $H_{\gamma_{0} \delta_{0}}^{*}=\left(\varepsilon_{1} M_{1}, \cdots, \varepsilon_{j-1} M_{j+1}, M_{j-1}, \cdots, M_{n}\right)$ and $K^{*}=\left\{\left\{H_{\gamma \delta}^{*}\right\}\right\}$. Clearly $K^{*} \in \mathscr{K}^{+}$, (positive parts of $K^{*}$ and $K$ correspond, one to many depending on the choice in $M_{j,}$ and, in any corresponding positive parts $P^{*}, P$ of $H_{\gamma_{0} \delta_{n}}$ the elements of $\bar{P}^{*}$ are greater than the corresponding elements of $\bar{P}$ ). Let $N_{\gamma_{0} \delta_{0}}^{*}, N_{\gamma_{0} \delta_{0}}$ be corresponding negative parts of $H_{\gamma_{0} \delta_{0}}$ and $u+v$, with $u \in \varepsilon_{1} M_{1}+\cdots+\varepsilon_{j-1} M_{j-1}$ and $v \in M_{j+1}+\cdots+M_{n}$, an element of $\bar{N}_{\gamma_{0} \delta_{0}}^{*}$. Because $g \perp v$ and $g \perp M_{j}$, $g \wedge(u+v)^{-}=g \wedge u^{-}=g \wedge(u-m+v)^{-}\left(m \in M_{j}\right)$. As in Case 2 we can conclude that

$$
g^{*}=g=\sup \left\{g \wedge v^{-}: v \in \bar{N}^{*}\right\}
$$

for every negative subsystem of $K^{*}$. 
CASE 4. $\varepsilon_{j}=-1$ and there exists $m \in M_{j}$ such that $g \wedge m \neq 0$. Choose such an $m$, put $g^{*}=g \wedge m, H_{\gamma \delta}^{*}=H_{y \delta}\left((\gamma, \delta) \neq\left(\gamma_{0}, \delta_{0}\right)\right)$ and $H_{\gamma_{0} \delta_{0}}^{*}$ $=\left(-\{m\}, \varepsilon_{1}\left(m+M_{1}-m\right), \cdots, \varepsilon_{j-1}\left(m+M_{j-1}-m\right), M_{j+1}, \cdots, M_{n}\right)$. A positive part $P^{*}$ of $H_{\gamma_{0} \delta_{0}}^{*}$ corresponds, in the usual way, to a positive part $P$ of $H_{\gamma_{0} \delta_{0}}$ where we fix $m$ in $M_{j}$ and we have, taking conjugations into account, $\bar{P}^{*}=\bar{P}$. It follows that $K^{*}=\left\{\left\{\boldsymbol{H}_{\gamma \delta}^{*}\right\}\right\} \in \mathscr{K}+$.

If $N_{\gamma_{0} \delta_{0}}^{*}$ is a negative part of $H_{\gamma_{0} \delta_{0}}^{*}$ and $N_{\gamma_{0} \delta_{0}}$ the corresponding negative part of $H_{\gamma_{0} \delta_{0}}$ we have $\bar{N}_{\gamma_{0} \delta_{0}}^{*} \subset \bar{N}_{\gamma_{0} \delta_{0}}$, while if $x \in \bar{N}_{\gamma_{0} \delta_{0}} \sim \bar{N}_{\gamma_{0} \delta_{0}}^{*}, x=u-m^{\prime}+v$ with $m^{\prime} \in M_{j} \sim\{m\}, u \in \varepsilon_{1} M_{1}+\cdots+\varepsilon_{j-1} M_{j-1}, v \in M_{j+1}+\cdots+M_{n}$. Since $v \perp g^{*}$ and $m^{\prime} \perp g^{*}$ we have $g^{*} \wedge\left(u-m^{\prime}+v\right)^{-}=g^{*} \wedge u^{-} \leqq g^{*} \wedge(u-m)^{-}$ $=g^{*} \wedge(u-m+v)^{-}$. Since $u-m+v \in \bar{N}_{\gamma_{0} \delta_{0}}^{*}$ we can conclude that

$$
g^{*}=\sup \left\{g \wedge v^{-}: v^{-} \in \bar{N}^{*}\right\}
$$

for every negative subsystem $N^{*}$ of $K^{*}$.

Repeating this argument a finite number of times we see that we can modify $g$ and $H_{\gamma_{0} \delta_{0}}$ so that $g$ remains positive or zero as the initial case may be, and so that

$$
H_{\gamma_{0} \delta_{0}}=\left(\varepsilon_{1} M_{1}, \cdots, \varepsilon_{k} M_{k}, M_{k+1}, \cdots, M_{r}\right)
$$

with $M_{1}, \cdots, M_{k}$ all singletons. Continuing we can assume that all the other $H_{y \delta}$ also have this form.

It follows that each $H_{y \delta}$ is its own unique positive part and $K$ is its own unique positive subsystem. If $h_{y \delta} \in \bar{H}_{y \delta}$ there is a negative part $N_{y \delta}$ of $H_{y \delta}$ such that $\left\{h_{y \delta}\right\}=\bar{N}_{y \delta}$. Define a negative subsystem $N$ of $K$ by $N=\left\{\left\{N_{y \delta}\right\}\right\}$. Then $\bar{N}=\left\{\vee_{\gamma} \wedge_{\delta} h_{y \delta}\right\}$ and $g=\sup \left\{g \wedge v^{-}: v \in \bar{N}\right\}$, which means $g \leqq\left(\vee_{\gamma} \wedge_{\delta} h_{y \delta}\right)^{-}$. Thus $g$ is a lower bound for $K^{-}$. Since $K$ is a positive part of $K \in \mathscr{K}^{+}$, $g \leqq \inf R^{-}=0$. Since our modification procedure never reduced $g$ to zero, unless it was already zero, we conclude that $L \in \mathscr{K}^{-}$as required.

The proof for $K+L \in \mathscr{K}^{-}$is similar so we omit it.

(ii) This part can be proved in the same way as part (i). The following argument is rather easier. From $L+K \in \mathscr{K}^{+}$and $K \in \mathscr{K}^{-}$we have, by Lemma 2.4 (ii) $L+K-L \in \mathscr{K}^{-}$so, by part (i) above, $-L \in \mathscr{K}^{-}$. This follows similarly if $K+L \in \mathscr{K}^{+}$.

Now, let $P=\left\{\left\{P_{\alpha \beta}\right\}\right\}$ be a positive subsystem of $L$, then, by (B),

$$
\begin{aligned}
\inf \bar{P}^{-} & =\inf \left[\bigwedge_{\alpha} \bigvee_{\beta}\left(-P_{\alpha \beta}\right)^{+}\right] \\
& =\inf \left[\bigvee_{f \in B_{\alpha}} \bigwedge_{\alpha \in A}\left(-P_{\alpha f(\alpha)}\right)^{+}\right] \\
& =0,
\end{aligned}
$$

because $\left\{\left\{-P_{\alpha f(\alpha)}: \alpha \in A\right\}: f \in X B_{\alpha}\right\}$ is a negative subsystem of $-L \in \mathscr{K}^{-}$. Thus $L \in \mathscr{K}^{+}$as required. 
Corollary 2.6. Any element of $\mathscr{K}^{+}$(respectively $\left.\mathscr{K}-, \mathscr{K}\right)$ can be cancelled

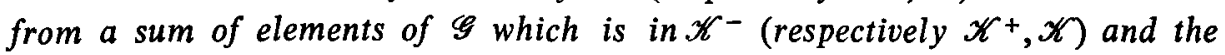
resulting sum is in $\mathscr{K}^{-}$(respectively $\left.\mathscr{K}^{+}, \mathscr{K}\right)$.

Proof. Lemma 2.5 applies directly to sums of the form $K+L, L+K$. Suppose $K \in \mathscr{K}^{+}$and $L+K+M \in \mathscr{K}^{-}$, we must show $L+M \in \mathscr{K}^{-}$. We have, by Lemma 2.4 (i),

$$
(L+M)+(L+K+M-M-L)=L+(M+(L+K+M)-M)-L \epsilon \mathscr{K}^{-} ;
$$

also, by Lemmas 2.3, 2.4,

$$
L+K+M-M-L=L+(K+(M-M))-L \in \mathscr{K}^{+} .
$$

Hence, by Lemma 2.5 (i), $L+M \in \mathscr{K}^{-}$.

The case $K \in \mathscr{K}^{-}$and $L+K+M \in \mathscr{K}^{+}$is similar and the results for $\mathscr{K}$ are clear.

The next step is to construct an equivalence relation on $\mathscr{G}$. We define

$$
\mathscr{R}=\left\{\left(L_{1}, L_{2}\right) \in \mathscr{G} \times \mathscr{G}: L_{1}-L_{2} \in \mathscr{K}\right\},
$$

Using Lemmas 2.3, 2.4 and Corollary 2.6 we have: (i) $(L, L) \in \mathscr{K}(L \in \mathscr{G})$; (ii) if $L_{1}-L_{2} \in \mathscr{K},\left(L_{2}-L_{1}\right)+\left(L_{1}-L_{2}\right)=L_{2}+\left(-L_{1}+L_{1}\right)-L_{2} \in \mathscr{K}$ and hence $L_{2}-L_{1} \in \mathscr{K}$; (iii) if $L_{1}-L_{2} \in \mathscr{K}, L_{2}-L_{3} \in \mathscr{K}$ then $L_{1}+\left(-L_{2}+L_{2}\right)$ $-L_{3} \in \mathscr{K}$ and hence $L_{1}-L_{3} \in \mathscr{K}$. It follows that $\mathscr{R}$ is an equivalence relation.

Let $G$ denote the set of $\mathscr{R}$-equivalence classes of $\mathscr{G}$ and, for $L \in \mathscr{G}$, let $\langle L\rangle$ denote the $\mathscr{R}$-equivalence class determined by $L$. If $g \in G$ we abbreviate $\left\langle\left\{\left\{\left(\left\{g^{+}\right\},-\left\{g^{-}\right\}\right)\right\}\right\}\right\rangle$to $\langle g\rangle$.

(The next two lemmas are identical in content with Theorems 3.2, 3.4 in my paper Free non-Abelian lattice groups, Math. Ann. 186 (1970), 249-262. They are proved here for completeness and because this paper was written first.)

LEMMA 2.7. With the natural definition $\left\langle L_{1}\right\rangle+\left\langle L_{2}\right\rangle=\left\langle L_{1}+L_{2}\right\rangle, G$ is an additive group.

Proof. Suppose $L_{1}^{*} \in\left\langle L_{1}\right\rangle, L_{2}^{*} \in\left\langle L_{2}\right\rangle$, and put $U=L_{1}+L_{2}-\left(L_{1}^{*}+L_{2}^{*}\right)$. We have

$$
U+L_{1}^{*}+L_{2}^{*}-L_{2}-L_{1}=L_{1}+\left(L_{2}+\left(-\left(L_{1}^{*}+L_{2}^{*}\right)+L_{1}^{*}+L_{2}^{*}\right)-L_{2}\right)-L_{1} \in \mathscr{K} .
$$

Cancelling $L_{2}^{*}-L_{2}$ and then $L_{1}^{*}-L_{1}$ we have $U \in \mathscr{K}$. Thus addition in $G$ is well defined. Associativity now follows from associativity in $\mathscr{G},\langle 0\rangle$ is the zero element (henceforth written 0 ) and Lemma 2.4 provides additive inverses.

Before our next definition we note that if $L \in \mathscr{K}^{+}\left(\mathscr{K}^{-}\right)$and $L^{*} \in\langle L\rangle$, then $L^{*}+(-L+L)=\left(L^{*}-L\right)+L \in \mathscr{K}^{+}$and by Corollary $2.6, L^{*} \in \mathscr{K}^{+}$. Now define $\left\langle L_{1}\right\rangle \geqq\left\langle L_{2}\right\rangle$ in $G$ to mean $L_{1}-L_{2} \in \mathscr{K}^{+}$. It is clear that this definition 
is independent of our choice of representatives from $\left\langle L_{1}\right\rangle,\left\langle L_{2}\right\rangle$ and is equivalent to any of $-L_{2}+L_{1} \in \mathscr{K}^{+},\left\langle L_{1}-L_{2}\right\rangle \subset \mathscr{K}^{+},\left\langle-L_{2}+L_{1}\right\rangle \subset \mathscr{K}^{+}$.

LEMMA 2.8. The relation $\geqq$ defined above is a partial ordering on $G$ under which $\bar{G}$ is a partially ordered group.

ProOF. Reflexivity, antisymmetry and transitivity are immediate. If $\langle L\rangle$ $\geqq\left\langle L^{*}\right\rangle$ and $M, N \in \mathscr{G}$ we have

$$
\begin{aligned}
\left\langle M+L+N-\left(M+L^{*}+N\right)\right\rangle & =\left\langle M+L+N-N-L^{*}-M\right\rangle \\
& =\left\langle M+L-L^{*}-M\right\rangle \\
& \subset \mathscr{K}^{+} .
\end{aligned}
$$

The ordering of $\bar{G}$ is therefore translation invariant. This completes the proof.

THEOREM 2.9. $\bar{G}$ is a lattice group in which, for

$$
\begin{gathered}
L=\left\{\left\{H_{\alpha \beta}: \beta \in B_{\alpha}\right\}: \alpha \in A\right\} \in \mathscr{G} \text { we have } \\
\langle L\rangle=\bigvee_{\alpha \in A \beta \in B_{-}}\left\langle\left\{\left\{H_{\alpha \beta}\right\}\right\}\right\rangle,
\end{gathered}
$$

and hence

$$
\left\langle L_{1}\right\rangle \vee\left\langle L_{2}\right\rangle=\left\langle L_{1} \vee L_{2}\right\rangle,\left\langle L_{1}\right\rangle \wedge\left\langle L_{2}\right\rangle=\left\langle L_{1} \wedge L_{2}\right\rangle\left(L_{1}, L_{2} \in \mathscr{G}\right) .
$$

Proof. Suppose $L_{1}, L_{2} \in \mathscr{G}$, then

$$
\left(L_{1} \vee L_{2}\right)-L_{1}=\left(L_{1}-L_{1}\right) \vee\left(L_{1}-L_{2}\right)
$$

Hence a positive subsystem $Q$ of $L_{1} \vee L_{2}-L_{1}$ can be written $R \vee S$ with $R$, $S$ positive subsystems of $L_{1}-L_{1}, L_{2}-L_{1}$ respectively. Because $L_{1}-L_{1} \in \mathscr{K}$ $\subset \mathscr{K}^{+}, \wedge \bar{Q}^{-}=\wedge(\bar{R} \vee \bar{S})^{-} \leqq \wedge \bar{R}^{-}=0$. Thus, $\left\langle L_{1} \vee L_{2}\right\rangle \geqq\left\langle L_{1}\right\rangle$ and similarly $\left\langle L_{1} \vee L_{2}\right\rangle \geqq\left\langle L_{2}\right\rangle$. Now suppose $\langle M\rangle \geqq\left\langle L_{1}\right\rangle,\langle M\rangle \geqq\left\langle L_{2}\right\rangle$. Considering $L_{1} \vee L_{2}-M$ we see that any negative subsystem $Q$ is of the form $Q=R \vee S$ with $R, S$ negative subsystems of $L_{1}-M, L_{2}-M$. Hence

$$
\wedge \bar{Q}^{+}=\wedge(\bar{R} \vee \bar{S})^{+}=\left(\wedge \bar{R}^{+}\right) \vee\left(\wedge S^{+}\right)=0
$$

It follows that $\left\langle L_{1} \vee L_{2}\right\rangle=\left\langle L_{1}\right\rangle \vee\left\langle L_{2}\right\rangle$.

Note that in this next part of the proof index sets are not omitted, thus $\left\{H_{\alpha \beta}\right\}$ is a singleton as is $\left\{\left\{H_{\alpha \beta}\right\}\right\}$. Taking $L$ as given we have, using $\left\langle L_{1} \vee L_{2}\right\rangle$ $=\left\langle L_{1}\right\rangle \vee\left\langle L_{2}\right\rangle$,

$$
\bigwedge_{\beta \in B_{-}}\left\langle\left\{\left\{H_{\alpha \beta}\right\}\right\}\right\rangle=-\bigwedge_{\beta \in B_{-}}\left\langle\left\{\left\{-H_{\alpha \beta}\right\}\right\}\right\rangle=-\left\langle\left\{\left\{-H_{\alpha \beta}\right\}: \beta \in B_{\alpha}\right\}\right\rangle=\left\langle\left\{\left\{H_{\alpha \beta}: \beta \in B_{\alpha}\right\}\right\}\right\rangle .
$$

Hence,

$$
\bigvee_{\alpha \in A} \bigwedge_{\beta \in B}\left\langle\left\{\left\{H_{\alpha \beta}\right\}\right\}\right\rangle=\bigvee_{\alpha \in A}\left\langle\left\{\left\{H_{\alpha \beta}: \beta \in B_{\alpha}\right\}\right\}\right\rangle=\left\langle\left\{\left\{H_{\alpha \beta}: \beta \in B_{\alpha}\right\}: \alpha \in A\right\}\right\rangle=\langle L\rangle .
$$


THEOREM 2.10. The natural map $g \rightarrow\langle g\rangle$ of $G$ into $\bar{G}$ is a group and order isomorphism of $G$ onto a dense sublattice group of $\bar{G}$. All suprema and inffima in $G$ are preserved in $\bar{G}$ and if $M \in \mathscr{D},\{\langle g\rangle: g \in M\}$ is a positive disjoint subset of $\bar{G}$ with supremum $\langle\{\{(M)\}\}\rangle$.

Proor. It is easy to check that

$$
\left\langle g_{1}+g_{2}\right\rangle=\left\langle g_{1}\right\rangle+\left\langle g_{2}\right\rangle\left(g_{1}, g_{2} \in G\right)
$$

and that $\langle g\rangle \geqq 0$ (in $\bar{G}$ ) if and only if $g \geqq 0$ (in $G$ ). This verifies the group and order isomorphism property.

Let $\langle L\rangle \in \bar{G}$, and suppose $\langle L\rangle \geqq 0,\langle L\rangle \neq 0$, then $L \in \mathscr{K}^{+}$and $L \notin \mathscr{K}^{-}$. Hence, there exists a negative subsystem $N$ of $L$ and $g \in G$ such that $g \geqq 0$, $g \neq 0$ and $g \leqq n^{+}(n \in \bar{N})$. Consider $Q=L-\{\{(\{g\})\}\}$. If $R$ is a positive subsystem of $Q$ then $R=S-\{\{(\{g\})\}\}$ for some positive subsystem $S$ of $L$ and $\bar{R}=S-g$. Because $L \in \mathscr{K}^{+}, \inf \bar{R}^{-} \leqq g+\inf \bar{S}^{-}=g$.

Let $H=\left(\varepsilon_{1} M_{1}, \cdots, \varepsilon_{n} M_{n}\right) \in \mathscr{H}$ and let $H_{1}$ be a positive and $H_{2}$ a negative part of $H$. Since $H_{1}$ fixes singleton subsets of the $M_{i}$ with $\varepsilon_{i}=-1$ and $H_{2}$ fixes singleton subsets of the $M_{i}$ with $\varepsilon_{i}=1$, there is a unique element $u \in \bar{H}_{1} \cap \bar{H}_{2}$. It follows that there exists a unique element $v \in S \cap \bar{N}$. Then $g \leqq v^{+}$and $(v-g)^{-}$ $\leqq v^{-}$. Hence

$$
\inf \bar{R}^{-} \leqq g \wedge v^{-} \leqq v^{+} \wedge v^{-}=0 .
$$

It follows that $\langle L\rangle-\langle g\rangle \geqq 0$. Because $g \geqq 0$ and $g \neq 0,\langle g\rangle \neq 0$ and $\langle g\rangle \geqq 0$. Thus the image of $G$ is dense in $\bar{G}$ as required.

By [2, Lemma 10] or [7, Lemma 2.3] $g \rightarrow\langle g\rangle$ preserves all suprema and infima in $G$. In particular the image of $G$ is a sublattice group of $G$.

Now let $M \in \mathscr{D}$. By the density property just proved $\{\langle g\rangle: g \in M\}$ is a positive disjoint subset of $\bar{G}$. It is easy to check that $\langle\{\{(M)\}\}\rangle \geqq\langle g\rangle(g \in M)$. Suppose $L=\left\{\left\{H_{\alpha \beta}: \beta \in B_{\alpha}\right\}: \alpha \in A\right\}$, and $\langle L\rangle \geqq\langle g\rangle(g \in M)$.

If $S$ is a positive subsystem of $L-\{\{(M)\}\}$, then $S=\left\{\left\{R_{\alpha \beta}-\left\{\left(g_{z \beta}\right)\right\}: \beta \in B_{\alpha}\right\}\right.$ : $\alpha \in A\}$ with $R_{\alpha \beta}$ a positive part of $H_{\alpha \beta}$ and $g_{\alpha \beta} \in M\left(\beta \in B_{\alpha}, \alpha \in A\right)$. Observe that if $M_{1}=\left\{g_{\alpha \beta}: \beta \in B_{\alpha}, \alpha \in A\right\}$ then $M_{1}$ is a finite subset of $M$ and $S$ is a positive part of $T=L-\left\{\left\{\left(M_{1}\right)\right\}\right\}$. Since $\langle L\rangle \geqq\langle g\rangle(g \in M)\langle L\rangle \geqq\left\langle\vee M_{1}\right\rangle$ and $T_{1}=L$ $-\left\{\left\{\left(\left\{\bigvee M_{1}\right\}\right)\right\}\right\} \in \mathscr{K}^{+}$. Consider $K=\left\{\left\{\left(\left\{\bigvee M_{1}\right\}\right)\right\}\right\}-\left\{\left\{\left(M_{1}\right)\right\}\right\}=\left\{\left\{\left(\left\{\bigvee M_{1}\right\}\right)\right.\right.$, $\left.\left.\left.-M_{1}\right)\right\}\right\} \in \mathscr{G}$. We see that $K$ is its own unique negative subsystem and that $R=\left(\bigvee M_{1}\right)-M_{1}$. Hence $\wedge R^{+}=\wedge\left(\bigvee M_{1}-M_{1}\right)^{+}=\left(\bigvee M_{1}-\bigvee M_{1}\right)^{+}=0$. A positive subsystem of $K$ is the form $R=\left\{\left\{\left(\left\{\bigvee M_{1}\right\},-\{m\}\right)\right\}\right\}$ with $m \in M_{1}$. Clearly $\bar{R}=\left\{\bigvee M_{1}-m\right\}$ and $\bar{R}^{-}=\{0\}$. Thus $K \in \mathscr{K}$. By Lemma 2.3,

$$
L-\left\{\left\{\left(\left\{\bigvee M_{1}\right\}\right)\right\}\right\}+\left\{\left\{\left(\left\{\bigvee M_{1}\right\}\right)\right\}\right\}-\left\{\left\{\left(M_{1}\right)\right\}\right\}=T_{1}+K \in \mathscr{K}^{+}
$$

and by Lemma 2.4 and Corollary 2.6 we may cancel the two middle terms to get $T=L-\left\{\left\{\left(M_{1}\right)\right\}\right\} \in \mathscr{K}^{+}$. This gives inf $\bar{S}^{-}=0$ for any positive subsystem of $L-\{\{(M)\}\}$ so that $\langle L\rangle \geqq\langle\{\{(M)\}\}\rangle$ which completes our proof. 


\section{The lateral completion of a lattice group}

The construction of Section 2 provides a lattice group $\bar{G}$ containing a dense copy of $G$ and suprema for every positive disjoint subset of $G$ and is, in fact, generated by these suprema. We continue by transfinite induction as follows.

Define $G(0)=G, G(1)=\overline{G(0)}$ and $\pi(1,0)$ to be the embedding $G(0) \rightarrow G(1)$ defined by $g \rightarrow\langle g\rangle$. Suppose that for all ordinals $\mu$, v such that $\mu \leqq v<\lambda$ we have constructed lattice groups $G(\mu)$ and lattice group isomorphisms $\pi(v, \mu)$ of $G(\mu)$ into $G(v)$ such that

(i) $\pi(\mu, \mu)$ is the identity on $G(\mu)$;

(ii) $\pi(v, \mu) \pi(\mu, \tau)=\pi(v, \tau)$ if $(\tau \leqq \mu \leqq v)$;

(iii) $G(\mu+1)=\overline{G(\mu)}$ and $\pi(\mu+1, \mu)$ is the associated natural embedding if $\mu+1<\lambda$.

Then, if $\lambda=v+1$ for some $v$, take $G(\lambda)=\overline{G(v)}, \pi(\lambda, v)$ the associated natural embedding, $\pi(\lambda, \lambda)$ the identity on $G(\lambda)$ and, for $\mu<v$ put $\pi(\lambda, \mu)$ $\pi(\lambda, v) \pi(v, \mu)$.

If $\lambda$ is a limit ordinal we take $G(\lambda)$ to be the direct limit of the $G(\mu)$ with $\mu<\lambda$. We may represent $G(\lambda)$ as follows (Conrad (1969)). $G(\lambda)$ is the subset of the large cardinal product $\Pi_{\mu<\lambda}(\mu)$ consisting of elements $g$ such that $g=0$ or if $\mu$ is the least ordinal such that $g(\mu) \neq 0$ then $g(\mu) \notin \bigcup_{v<\mu} \pi(\mu, v) G(v)$ and $g(v)=$ $\pi(v, \mu) g(\mu)$ if $\mu \leqq v<\lambda$. Any non-zero component of $g$ completely determines $g, g \geqq 0$ if $g=0$ or if $g$ has a positive component, and the sum of two elements $g$ and $h$ is found by taking an ordinal $\mu$ such that $g(\mu) \neq 0, h(\mu) \neq 0$ and determining $g+h$ by $(g+h)(\mu)=g(\mu)+h(\mu)$. We determine $g \vee h$ and $g \wedge h$ similarly. By Conrad (1969), Theorem 3.1, $G(\lambda)$ is a lattice group. We take $\pi(\lambda, \lambda)$ to be the identity on $G(\lambda)$ and determine each $\pi(\lambda, \mu)$ by

$$
(\pi(\lambda, \mu) g)(\mu)=g \quad(g \in G(\mu), \mu<\lambda) .
$$

Condition (ii) is easily verified from the representation of $G(\lambda)$ and (iii) is vacuous.

THEOREM 3.1. For ordinals $\lambda, \mu$ such that $\mu<\lambda, \pi(\lambda, \mu) G(\mu)$ is a dense sublattice group of $G(\lambda)$ and, if $M$ is a positive disjoint subset of $G(\mu)$, then $\pi(\lambda, \mu) M$ has a supremum in $G(\lambda)$.

Proof. For the first result we use transfinite induction. For $\lambda=v+1>v \geqq \mu$ we have $G(\lambda)=\overline{G(v)}$ and $\pi(v, \mu) G(\mu)$ is dense in $G(v)$. Because $\pi(v+1, v) G(v)$ is dense in $\overline{G(v)}$ we have

$$
\pi(v+1, v) \pi(v, \mu) G(\mu)=\pi(\lambda, \mu) G(\mu)
$$

is dense in $G(\lambda)$. If $\lambda$ is a limit ordinal, $g \in G(\lambda)$ and $g>0$ then $g(v)>0$ for some $v$ such that $\mu \leqq v<\lambda$. By the inductive hypothesis $\pi(v, \mu) G(\mu)$ is dense in $G(v)$ so there exist $h \in G(\mu)$ such that $0<\pi(v, \mu) h \leqq g(v)$. It follows that $0<\pi(\lambda, \mu) h \leqq g$. 
For the second result we have, by construction, that $\pi(\mu+1, \mu) M$ has a supremum, say $m$, in $G(\mu+1)$ and, by Theorem $2.10, \pi(\lambda, \mu+1) m$ is the supremum, in $G(\lambda)$, of $\pi(\lambda, \mu+1) \pi(\mu+1, \mu) M=\pi(\lambda, \mu) M$.

Corollary 3.2. We have $\pi(\lambda+1, \lambda) G(\lambda)=G(\lambda+1)$, if and only if $G(\lambda)$ is laterally complete.

Proof. By Theorem 3.1, if $M$ is a positive disjoint subset of $G(\lambda)$ then $\pi(\lambda+1, \lambda) M$ has a supremum, say $m_{1}$, in $G(\lambda+1)$. By hypothesis $m_{1}=\pi(\lambda+$ $1, \lambda) m$ for some $m \in G(\lambda)$. It follows that $m$ is the supremum of $M$ in $G(\lambda)$.

The converse follows from Theorem 2.10 and density of $\pi(\lambda+1, \lambda) G(\lambda)$ in $G(\lambda+1)$.

THEOREM 3.3. For any lattice group $G$ there exists an ordinal $\lambda$ such that $G(\lambda)$ is laterally complete.

Proof. Let $B$ be the cardinal of $G$ (considered as a set); note that $B$ is infinite (except in the trivial case $G=\{0\}$ ); and let $\lambda$ be the least ordinal with cardinal strictly greater than $B$. We show that $G(\lambda)$ is laterally complete.

Suppose $M=\left\{g_{\alpha}: \alpha \in A\right\}$ ) is a positive disjoint subset of $G(\lambda)$. For each $\alpha \in A$ there exists $h_{\alpha} \in G$ such that $0<\pi(\lambda, 0) h_{\alpha} \leqq g_{\alpha}$. It follows that $h_{\alpha} \neq h_{\beta}$ if $\alpha \neq \beta$ and hence that $A$ has cardinal at most $B$. Note that $\lambda$ is a limit ordinal and for each $\alpha \in A$ let $\mu_{\alpha}$ be the least ordinal such that $g_{\alpha} \in \pi(\lambda, \mu) G(\mu)$. Then $U=\left\{\mu_{\alpha}: \alpha \in A\right\}$ is a set of ordinals of cardinal $B$, at most, and bounded above by $\lambda$. Because $\mu_{\alpha}<\lambda, \mu_{\alpha}$ has cardinal $B$ at most and hence, if $v$ is the supremum of $U, v$ has cardinal at most $B \times B=B$. Thus $v<\lambda$. Hence, for each $\alpha \in A$, there exists a unique $k_{\alpha} \in G(v)$ such that $g_{\alpha}=\pi(\lambda, v) k_{\alpha}$. It follows that $K=\left\{k_{\alpha}: \alpha \in A\right\}$ is a positive disjoint subset of $G(v)$ and, by Theorem 3.1, $M=\pi(\lambda, v) K$ has a supremum in $G(\lambda)$. Thus $G(\lambda)$ is laterally complete.

Let $\lambda$ now be the least ordinal such that $G(\lambda)$ is laterally complete. If, for each $v \leqq \lambda$ we identify $G(v)$ with $\pi(\lambda, v) G(v)$ we have inclusions

$$
G=G(0) \subset \cdots \subset G(\lambda)
$$

with each inclusion strict, $G(v+1)$ the sub lattice group of $G(\lambda)$ generated by the suprema of all positive disjoint subsets of $G(v)$ and $G(v)=\bigcup_{\mu<v} G(\mu)$ when $v$ is a limit ordinal. If $K$ is a laterally complete sub lattice group of $G(\lambda)$ such that $G \leqq K$ it follows by transfinite induction that $G(v) \subset K(v \leqq \lambda)$ and hence that $K=G(\lambda)$. Thus $G(\lambda)$ is a lateral completion of $G$.

LEMMA 3.4. Let $G$ be a lattice group, $K$ a laterally complete lattice group and $i: G \rightarrow K$ a lattice group isomorphism. If $i G$ is dense in $K$, then $i$ can be lifted to a lattice group isomorphism of $G$ into $K$. 
Proof. Suppose $L=\left\{\left\{H_{\alpha \beta}: \beta \in B_{\alpha}\right\}: \alpha \in A\right\} \in \mathscr{G}$ wilu

$$
H_{\alpha \beta}=\left(\varepsilon(\alpha, \beta)_{1} M(\alpha, \beta)_{1}, \cdots, \varepsilon(\alpha, \beta)_{n(\alpha, \beta)} M(\alpha, \beta)_{n(\alpha, \beta)}\right) .
$$

Because $i G$ is dense in $K$ and $K$ is laterally complete, $i M(\alpha, \beta)_{j}$ is a positive disjoint subset of $K$ and $\bigvee_{K} i M(\alpha, \beta)_{j}$ exists. Define $h_{\alpha \beta} \in K$ by $h_{\alpha \beta}=\varepsilon(\alpha, \beta)_{1} \bigvee_{K} i M(\alpha, \beta)_{1}$ $+\cdots+\varepsilon(\alpha, \beta)_{n(\alpha \beta)} \bigvee_{K} i M(\alpha, \beta)_{n(\alpha \beta)}$ and put $\theta\langle L\rangle=\bigvee_{\alpha} \wedge_{\beta} h_{\alpha \beta} \in K$. To verify that $\theta$ is well defined it is sufficient to show that if $L \in \mathscr{K}, \vee_{\alpha} \wedge_{\beta} h_{\alpha \beta}=0$. For this, suppose $N=\left\{\left\{N_{\alpha \beta}\right\}\right\}$ is a negative subsystem of $L$. Define $n_{\alpha \beta} \in K$ by analogy with the definition of $h_{\alpha \beta}$ above. Then,

$$
n_{\alpha \beta}=\bigwedge_{\mathbf{K}}\left\{i g: g \in \bar{N}_{\alpha \beta}\right\}
$$

and, if $\mathscr{N}_{\alpha \beta}$ denotes the set of all negative parts of $H_{\alpha \beta}$,

$$
h_{\alpha \beta}=\bigvee_{K}\left\{\bigwedge_{K}\left\{i g: g \in \bar{N}_{\alpha \beta}\right\}: N_{\alpha \beta} \in \mathscr{N}_{\alpha \beta}\right\} \text {. }
$$

Write $\mathscr{N}$ for the set of all negative subsystems of $L$, then

Hence,

$$
\begin{aligned}
& \bigvee_{\alpha} \bigwedge_{\beta} h_{\alpha \beta}=\bigvee_{\alpha} \bigwedge_{\beta} \bigvee_{K}\left\{\bigwedge_{K}\left\{i g: g \in \bar{N}_{\alpha \beta}\right\}: N_{\alpha \beta} \in \mathscr{N}_{\alpha \beta}\right\} \\
& \quad=\sup _{K}\left\{\inf _{\mathbf{K}}\left(\bigvee_{\alpha} \bigwedge_{\beta} i \bar{N}_{\alpha \beta}\right):\left\{\left\{N_{\alpha \beta}\right\}\right\} \in \mathscr{N}\right\}=\sup _{K}\left\{\inf _{\mathbf{K}} i \bar{N}: N \in \mathscr{N}\right\} .
\end{aligned}
$$

$$
\left(\bigvee_{\alpha} \bigwedge_{\beta} h_{\alpha \beta}\right)^{+}=\sup _{\mathbf{K}}\left\{\inf _{\mathbf{K}} i \bar{N}^{+}: N \in \mathscr{N}\right\}
$$

Because $i G$ is dense in $K$ and $L \in \mathscr{K}^{-}$,

$$
\inf _{K} i \bar{N}^{+}=i \inf _{K} \bar{N}^{+}=0 .
$$

Thus $\bigvee_{\alpha} \bigwedge_{\beta} h_{\alpha \beta} \leqq 0$. Similarly we have $\bigvee_{\alpha} \bigwedge_{\beta} h_{\alpha \beta} \geqq 0$. This shows that $\theta$ is well defined.

It is routine now to check that $\theta: G \rightarrow K$ is a lattice group homomorphism which lifts $i$. That $\theta$ is an isomorphism follows because $G$ is dense in $G$ and $\theta$ is 1-1 on $G$.

THEOREM 3.5. If $K$ is a lateral completion of $G^{\circ}$ then $G(\lambda)$ is isomorphic to $K$.

Proof. Suppose for each ordinal $\mu<v$ we have a lattice group isomorphism $i_{\mu}$ of $G(\mu)$ into $K$ which lifts the identity map $i$ of $G$ into $K$ and also Ifits each $i_{\mu^{\prime}}$, with $\mu^{\prime}<\mu$. If $v=\mu+1$ we use Lemma 3.4 to lift $i_{\mu}$ to $G(v)$. If $v$ is a limit ordinal and $g \in G(v)$ we pick a non-zero component $g(\mu)$ of $g$ and take $i_{v} g=i_{\mu} g(\mu)$. It is easily checked that this provides a lattice group homomorphism of $G(v)$ into $K$ and the one to one property follows because $G$ is dense in $K$. That $i_{\text {v }}$ lifts $i_{\mu}$ if $\mu<v$ is clear. 
This induction provides an embedding of $G(\lambda)$ into $K$ with $G$ a dense sub lattice group of $G(\lambda)$. It follows that suprema in $G(\lambda)$ agree with those in $K$ so that $G(\lambda)$ contains $G$ and is a laterally complete sub lattice group of $K$. Because $K$ is a lateral completion of $G$ we have the image of $G(\lambda)$ equal to $K$. This proves the theorem.

We summarise the results of this section in

THEOREM 3.6. Every lattice group has a unique lateral completion.

\section{Structure of the lateral completion}

In this section we shall look into properties of the lateral completion of $G$ which are inherited from $G$. The method of proof is usually by induction up the chain

$$
G=G(0) \subset \cdots \subset G(\lambda)
$$

where $G(\lambda)$ is the lateral completion.

First, if $G$ is abelian it is obvious from the construction of $G$ in section 2 that $G$ is abelian. The direct limit construction of $G(v)$ for limit ordinals also preserves the abelian property so we have verified the first part of the following.

Theorem 4.1. (Conrad (1969), Theorem 2.5). If $G$ is abelian, then so its lateral completion. If $G$ is also divisible, then so is its lateral completion.

Proof. We have already shown that $G(\lambda)$ is abelian. The proof of divisibility follows exactly as in Conrad (1969).

Recall that in a lattice group $G$ a polar subgroup is a subgroup of the form

$$
M^{\prime}=\{x:|x| \wedge|m|=0 \quad(m \in M)\}
$$

for some non-empty subset $M$ of $G$. All the material we need about polar subgroups can be found in Bernau (1965). We also recall that a lattice group $G$ is said to be representable if for all $a, x \in G, a \wedge(-x+a+x)=0$ implies $a=0$. This concept precedes, see for example Lorenzen (1969), and is equivalent to orthocommutativity which was defined in Bernau (1965) to mean that all polar subgroups of $\mathbf{G}$ are normal (suppose $a \wedge b=0$, put $u=a \wedge(x+b-x)$ and consider $u \wedge(-x+u+x)$.

Our next result is due to Conrad (1969), Theorem 2.8. The proof gives a considerable simplification of Conrad's and also contains his Lemmas 2.6, 2.7.

THEOREM 4.2. If $G$ is representable, then so is the lateral completion of $G$.

PROof. We begin with the observation, valid in any lattice group, that

$$
\begin{array}{r}
-x \vee y+c+x \vee y=(-x \vee y+c+x) \vee(-x \vee y+c+y) \\
\leqq(-x+c+x) \vee(-y+c+y),
\end{array}
$$

and similarly, 


$$
x \vee y+c-x \vee y \leqq(x+c-c-x) \vee(y+c-y) \text {. }
$$

Hence (compare Conrad's Lemma 2.6), if $b \wedge(-x+c+x)=0$ and $b \wedge(-y+$ $c+y)=0$, then

$$
b \wedge(-x \vee y+c+x \vee y)=0=b \wedge(-x \wedge y+c+x \wedge y) .
$$

To prove the lateral completion representable it is sufficient to show that $G(1)$ is representable. By our remarks above it is sufficient to show that if $b, \mathrm{c} \in G(1)$ and $b \wedge c=0$ then $b \wedge(-h+c+h)=0$ for all $h \in G(1)$ of the form $h=\varepsilon_{1} u_{1}+\cdots+\varepsilon_{n} u_{n}$ where for each $i, \varepsilon_{i}= \pm 1$ and $u_{i}=\vee M_{i}$ for a positive disjoint subset $M_{i}$ of $G$. For this it is clearly sufficient to consider $n=1$ and $h= \pm \vee M$. Now

and

$$
b \wedge(\bigvee M+c-\bigvee M)=\bigvee_{m \in M}(b \wedge(m+c-\bigvee M))
$$

$$
b \wedge(m+c-\vee M) \leqq b \wedge(m+c-m) \text {. }
$$

Thus it is sufficient to show that if $b, c \in G(1), m \in G$ and $b \wedge c=0$, then $b \wedge(m+c-m)=0$.

Suppose $g \in G$ and $g \leqq b \wedge(m+c-m)$, then $g \wedge(-m+g+m)$ $\leqq b \wedge c=0$. Because $G$ is representable, $g \leqq 0$ and, because $G$ is dense in $G(1), b \wedge(m+c-m)=0(m \in G)$. This completes the proof.

Suppose temporarily that $G$ is archimedean. Then, $G$ is abelian and certainly representable. In Bernau (1966) we constructed an orthocompletion for a representable lattice group which, among other things, is laterally complete and contains a densely embedded copy of $G$. It follows from Lemma 3.4, as in the proof of Theorem 3.5, that the lateral completion, $G(\lambda)$, can be embedded in the orthocompletion. Because $G$ is archimedean so is the orthocompletion Bernau (1966), page 125, Remark 1 and hence the lateral completion of an archimedean lattice group is archimedean. In fact for archimedean lattice groups the lateral completion is the orthocompletion, this is proved by Veksler and Geiler (1972). Conrad (1969), Theorem 3.5 constructs the lateral completion of a representable lattice group and shows, as a corollary, that the Archimedean property is preserved. We give yet another proof based on our construction.

THEOREM 4.3. The lateral completion of an archimedean lattice group $G$ is archimedean.

Proof. It is again sufficient to consider $G(1)$. Suppose that $h_{1}, h_{2} \in G(1)$ and

$$
n h_{1} \leqq h_{2} \quad(n=1,2, \cdots),
$$

and that $h_{1}>0$. By construction of $G(1)$ there exists a finite collection $M_{1}, \cdots, M_{k}$ of positive disjoint subsets of $G$ such that, in $G(1)$ 


$$
h_{2} \leqq \bigvee M_{1}+\cdots+\bigvee M_{k} .
$$

Because $G$ is dense in $G(1)$ and $h_{1}>0$ there exists $g \in G$ such that $0<g<h_{1}$. Thus we have

$$
0<n g \leqq \vee M_{1}+\cdots+\bigvee M_{k} \quad(n=1,2, \cdots) .
$$

We show that this is impossible using induction on $k$.

If $k=1$ and $m \in M=M_{1}$ we have $m \wedge(\vee M-m)=0$ and hence

$$
\begin{gathered}
n(g \wedge m) \wedge(\vee M-m)=0 \quad \text { so that } \\
0 \leqq n(g \wedge m) \leqq m \quad(n=1,2, \cdots) .
\end{gathered}
$$

Because $G$ is archimedean $g \wedge m=0(m \in M)$ and $g=\bigvee(g \wedge m)=0$.

Now suppose the result true for $1 \leqq k<p$ and suppose

$$
0<n g \leqq \vee M_{1}+\cdots+\vee M_{p} \quad(n=1,2, \cdots) .
$$

Then, there exists a positive integer $n_{1}$ such that

$$
n_{1} g \text { 表 } \vee M_{p}
$$

Hence there exists $g_{1} \in G$ such that

$$
0<g_{1} \leqq\left(n_{1} g-\bigvee M_{p}\right)^{+} .
$$

For each positive integer $n$

$$
\begin{aligned}
0<n g_{1} & \leqq\left(n n_{1} g-n \bigvee M_{p}\right)^{+} \\
& \leqq\left(n n_{1} g-\bigvee M_{p}\right)^{+} \\
& \leqq \vee M_{1}+\cdots+\bigvee M_{p-1}
\end{aligned}
$$

By our inductive hypothesis this is impossible. It follows that $G(1)$ is archimedean.

\section{The radicals of the lateral completion}

Let us now recall some definitions as given, say, in Conrad (1964), (1969), or Byrd and Lloyd (1967). Let $M$ be a solid subgroup of a lattice group $G$. We say that $M$ is prime, closed, a value of $g$ (respectively) if $a \wedge b=0$ implies $a \in M$ or $b \in M, \mathrm{P} \subset M^{+}$and $\vee P$ exists in $G$ implies $\vee P \in M, M$ is maximal with respect to not containing $g$ (respectively). Further we call $M$ regular if $M$ is a value of some element $g \in G$. and essential if $M$ is regular and for some $h \in G, M>R_{h}$ where $R_{h}$ is the subgroup of $G$ generated by all the values of $h$. It is easily seen that regular subgroups are prime. The analogous concepts for $l$-ideals (solid normal subgroups) of $G$ are also defined, but note that regular $l$-ideals need not be prime.

Three types of radicals have been defined for a lattice group. These are: the radical (Conrad (1964)), $R(G)=\bigcap\left\{R_{g}: g \in G, g \neq 0\right\}$, where $R_{g}$ is the subgroup 
generated by all values of $g$; the distributive radical, Byrd and Lloyd (1967), $D(G)$ equal to the intersection of all closed prime subgroups of $G$; and the ideal radical, Conrad (1964), $L(G)=\bigcap\left\{\left(L_{g}: g \in G, g \neq 0\right\}\right.$ where $L_{g}$ is the $l$-ideal generated by all ideal values of $g$. It is known that each of these radicals is an $l$-ideal of $G$, that

$$
L(G) \subset D(G) \subset R(G),
$$

that $G$ is completely distributive if and only if $D(G)=\{0\}$, and that $R(G)(L(G))$ is the intersection of all essential subgroups ( $l$-ideals) of $G$. By a result of Byrd (1969), Proposition 4.1, essential subgroups are closed so it follows that $R(G)$ is closed (this is obvious for $D(G)$ ).

We now quote two results of Conrad (1969) in a strengthened form due to Byrd and Lloyd (1969), Lemmas 1 and 2. As foreshadowed in $\S 1$ we now use the $G(\mu)$ notation interchangeably for $\mathscr{L}$-completions of $G$ and for the lateral completion of $G$.

LeMmA 5.1. Let $G$ be a lattice group, $H=G(\lambda)$ an $\mathscr{L}$-completion of $G$ and suppose that $C$ is a solid subgroup of $G(1) \subset H$ such that:

(1) $C \cap G$ is prime in $G$;

(2) if $M$ is a positive disjoint subset of $C \cap G$, then $\vee M \in C$.

Then (i) $C+G=G(1)$, and (ii) $C$ is prime in $G(1)$.

Now suppose $B$ is a solid subgroup of $G$ and let $C$ be the solid subgroup of $G(1)$ generated by all elements of the form $\bigvee M$ where $M$ is a positive disjoint subset of $B$. We shall use the notation $C=\bigvee_{G(1)}(\bigvee M)(M \in \mathscr{D}(B))$.

LEMMA 5.2. If $B$ is a closed prime subgroup of $G$ and $C=\bigvee_{G(1)}(\bigvee M)$ $(M \in \mathscr{D}(B))$, then:

(i) if $x \in C$ and $x>0$, then $x \leqq \bigvee M_{1}+\cdots+\bigvee M_{n}$ where $M_{i} \in \mathscr{D}(B)$ $(i=1,2, \cdots, n)$ and hence $x \leqq \bigvee P$ for some subset $P$ of $B^{+}$;

(ii) $C \cap G=B$;

(iii) $C+G=G(1)$ and $C$ is prime in $G(1)$;

(iv) $C$ is closed in $G(1)$;

(v) if in addition, $g \in G, g>0$ and $B$ is a value of $g$ in $G$, then $C$ is a value of $g$ in $G(1)$.

We shall extend these results a little further (compare Byrd and Lloyd (1969), parts of the proof of Theorem 1). Take $B$ a closed prime subgroup of $G, H=G(\lambda)$ an $\mathscr{L}$-completion of $G$, and define subgroups $C(\mu)$ of $G(\mu)$ for each ordinal $\mu \leqq \lambda$ by induction as follows,

$$
\begin{aligned}
C(0) & =B \\
C(\mu+1) & =\vee_{G(\mu+1)}(\vee M) \quad(M \in \mathscr{D}(C(\mu))
\end{aligned}
$$

and, for limit ordinals $v \leqq \lambda$,

$$
C(v)=\bigcup_{\mu<v} C(\mu)
$$


LEMMA 5.3. With $B$ and $C(\mu)$ defined as above we have, for each ordinal $\mu \leqq \lambda$ :

(i) if $x \in C(\mu)$ and $x>0$ then $x=\vee\left\{x \wedge g: g \in B^{+}\right\}$;

(ii) $C(\mu) \cap G=B$;

(iii) $C(\mu)+G=G(\mu)$ and $C(\mu)$ is prime in $G(\mu)$;

(iv) $C(\mu)$ is closed in $G(\mu)$;

(v) if in addition $g \in G, g>0$ and $B$ is a value of $g$, then $C(\mu)$ is a value of $g$ in $G(\mu)$.

Proof. We preceed by induction on $\mu$. Suppose the result is valid for all ordinals $v<\mu$. If $\mu=v+1$ then Lemma 5.2 proves (ii), (iii), (iv) and (v) while (i) follows from Lemma 5.2 (i) since we have, for non-zero $x \in C(\mu)^{+}, x \leqq \vee P$ for some subset of $P$ of $C(v)^{+}$. Thus

$$
\begin{aligned}
x & =\bigvee\{x \wedge p: p \in P\}=\bigvee\left\{x \wedge \vee\left\{p \wedge g: g \in B^{+}\right\}: p \in P\right\} \\
& =\bigvee\left\{x \wedge p \wedge g: g \in B^{+}, p \in P\right\} \leqq \bigvee\left\{x \wedge g: g \in B^{+}\right\} \leqq x .
\end{aligned}
$$

If $\mu$ is a limit ordinal we have $C(\mu)=\bigcup_{\nu<\mu} C(v)$ and $G(\mu)=\bigcup_{v<\mu} G(v)$. Parts (i) and (ii) follow immediately as does the equality $C(\mu)+G=G(\mu)$. If $a, b \in G(\mu), a \wedge b=0$ and $a \notin C(\mu)$ then $a, b \in G(v)$ for some $v<\mu$ and hence, $b \in C(v) \subset C(\mu)$. This proves (iii).

To prove (iv) suppose that $P \subset C(\mu)^{+}$and $c=\vee P$ exists in $G(\mu)$. By (iii) we have $c=x+g$ with $x \in C(\mu), g \in G$. Because $c \geqq 0$ we have $x \geqq-g$ and hence, $g^{-} \in C(\mu)$. Thus we may assume $g \geqq 0$. Then we have $g=g \vee 0=(-x+c) \vee 0$ $=\bigvee\left\{(-x+p)^{+}: p \in P\right\}$. Writing $Q=(-x+P)^{+}$we have $Q \in C(\mu)^{+}$and $g=\bigvee Q \in G$. Thus $g=\bigvee\{g \wedge q: q \in Q\}$. By (i) we have

$$
g=\bigvee\left\{g \wedge \vee\left\{q \wedge g^{*}: g^{*} \in B^{+}\right\}: q \in Q\right\} \leqq \vee\left\{g \wedge g^{*}: g^{*} \in B^{+}\right\} \leqq g
$$

Because $B$ is solid $g \wedge g^{*} \in B^{+}\left(g^{*} \in B^{+}\right)$and, because $B$ is closed, $g \in B$. Thus $c=x+g \in C(\mu)$ and $C(\mu)$ is closed.

For (v) suppose $h \in G(\mu), h \notin C(\mu)$, then, by (iii) $h=x+k$ with $x \in C(\mu)$, $k \in G$ and $k \notin G \cap C(\mu)=B$. Hence, if a solid subgroup $M$ of $G(\mu)$ properly contains $C(\mu)$ it contains an element of $G$ not in $B$. If $B$ is a value of $g$ in $G$ it follows that $g \in M$. Thus $C(\mu)$ is a value of $g$ in $G(\mu)$.

COROLLARY 5.4. With the hypotheses of Lemma 5.2 if $B$ is also a normal subgroup of $G$, then $C(\mu)$ is a normal subgroup of $G(\mu)$ for each $\mu \leqq \lambda$.

Proof. It is sufficient to show that $C=C(1)$ is normal in $G(1)$. If $h \in G(1)$ then $h=x+g$ with $x \in C, g \in G$. Thus $-h+C+h=-g+C+g$. If $M$ is a positive disjoint subset of $B,-g+M+g$ is a positive disjoint subset of $G$ and is contained in $B$ because $B$ is normal in $G$. The result follows.

THEOREM 5.5. If $H$ is an $\mathscr{L}$-completion of $G$, then $D(H) \cap G \subset D(G)$; if $H$ is the lateral completion of $G$ then $D(H) \cap G=D(G)$. 
Proof. If $g \notin D(G)$ there is a closed prime subgroup $B$ of $G$ such that $g \notin B$. Take $C(\lambda)$ as in Lemma 5.3. Then $C(\lambda)$ is a closed prime subgroup of $H$ and $C(\lambda) \cap G=B$. Thus $g \notin D(H) \cap G$.

If $H$ is the lateral completion of $G, g \in G$ and $g \notin D(H)$ there is a closed prime subgroup $C$ of $H$ such that $g \notin C$. Let $B=G \cap C$, then $B$ is a prime subgroup of $G$. If $L \subset B$ and $g_{0}=\bigvee_{G} L$ in $G$, then $g_{0}=\bigvee_{H} L$ because $G$ is dense in $H$. This gives $g_{0} \in C \cap G=B$, because $C$ is closed in $H$. Thus $B$ is a closed prime subgroup of $G, g \notin B \supset D(G)$. This proves our theorem.

COROLLARY 5.6. If $G$ is completely distributive so is its lateral completion.

Proof. Because $G$ is dense in its lateral completion $H, D(G)=\{0\}$ implies $D(H)=\{0\}$. The result follows.

This reverifies Byrd and Lloyd's Theorem 2 (1969) without the need to consider invariant sets of closed prime subgroups.

We turn now to the radical of an $\mathscr{L}$-completion of $G$. We recall Proposition 4.1 of Byrd (1967) in which it is shown that an essential subgroup of a lattice group is closed.

Lemma 5.7. Let $H=G(\lambda)$ be an $\mathscr{L}$-completion of $G$ and let $B$ be an essential subgroup of $G$. If $C=C(\lambda)$ is constructed from $B$ as in Lemma 5.3, then $C$ is an essential subgroup of $H$,

Proof. We consider two cases.

CASE 1. There exists a non-zero $h \in B^{+}$such that $R_{h} \subset B$. Let $P$ be a solid subgroup of $H$ such that $h \notin P$ and take $p \in P^{+}$. By Lemma 5.3 (iii) $p=c+g$ with $c \in C, g \in G$; then

$$
p=p^{+}=c^{+}-c^{+} \wedge g^{-}-c^{-} \wedge g^{+}+g^{+} .
$$

Thus, to show that $p \in C$ it is sufficient to show that if $g \in G^{+}, c \in C^{+}$and $0 \leqq-c+g \in P$, then $g \in B$.

Suppose under these circumstances that $g \notin B$. If $0 \leqq k<g$ and $k \in B$, then $-k+g \notin B$. Because $R_{h} \subset B$ and $h \in B$ no value of $h$ contains $(-k+g-h)^{+}$. It follows that $h \leqq n(-k+g-h)^{+}$for some positive integer $n$. Hence $0 \leqq(-k+g-h)^{-} \leqq h \leqq n(-k+g-h)^{+}$and $(-k+g-h)^{-}=0$. In other words $k \leqq g-h$ (and, in particular, $h<g$ ). By Lemma 5.3 (i), $c=\bigvee\left\{c \wedge g^{*}\right.$ : $\left.g^{*} \in B^{+}\right\}$and, because $c<g$ and $g \wedge g^{*} \leqq g-h\left(g^{*} \in B^{+}\right)$,

$$
c=\bigvee\left\{c \wedge g^{*} \wedge g: g^{*} \in B^{+}\right\} \leqq g-h .
$$

Thus $0<h \leqq-c+g \in P$ which contradicts our assumption that $h \notin P$. It follows that $g \in B, P \subset C$, every value of $h$ in $H$ is contained in $C$ and, by Lemma 5.3 (v), $C$ is an essential subgroup of $H$. 
CASE 2. For every $h \in G^{+}$such that $h>0$ and $R_{h} \subset B, h \notin B$. Choose one such $h$ and observe that $R_{h}=B$ and $B$ is the unique value of $h$ in $G$. If $g \in B^{+}$ and $g \wedge h \neq 0, R_{g \wedge h} \subset R_{h}=B$ and $g \wedge h \in B$. This is impossible so $g \wedge h=0$ and $B$ is the polar of $h$ in $G$ (so far we are repeating part of the proof of Byrd (1967), Proposition 4.1, Case 2, the rest is new). By Lemma 5.3 (v), $C$ is a value of $h$ in $H$ and hence contains the polar of $h$ in $H$. However, by Lemma 5.3 (i) if $u \in C$, $h \wedge|u|=0$, so $C$ is precisely the polar of $h$ in $H$. If $Q$ is any value of $h$ in $H$ then $Q$ contains the polar of $h$ in $H$, that is $Q \supset C$. Because $C$ is a value of $h, Q=C$. Thus $C$ is the unique value of $h$ in $H$ so again $C$ is an essential subgroup of $H$.

THEOREM. 5.8 If $H$ is an $\mathscr{L}$-completion of $G$ then $R(H) \cap G \subset R(G)$ and if $H$ is the lateral completion of $G, R(H) \cap G=R(G)$.

Proor. If $g \in G \sim R(G)$ there is an essential subgroup $B$ of $G$ such that $g \notin B$. Take $C$ as in Lemma 5.7, then $C$ is an essential subgroup of $H$ and $g \notin C$. Thus $R(H) \cap G \subset R(G)$.

Conversely, when $H$ is the lateral completion, if $g \in G \sim R(H)$ there is an essential subgroup $C$ of $H$ such that $g \notin C$. Take $u \in H^{+}$such that $u \neq 0$ and $R_{u} \cap C$. Because $G$ is dense in $H$, there is $h \in G$ such that $0<h \leqq u$. Clearly, in $G, R_{h} \subset G \cap C$. Hence, $G \cap C$ is an essential subgroup of $G$ and $g \notin G \cap C$. It follows that $R(H) \cap G=R(G)$.

COROLlary 5.9. If $H$ is the lateral completion of $G$ and $R(G)=\{0\}$, then $R(H)=\{0\}$.

Proor. This follows because $G$ is dense in $H$.

Whether $L(H) \cap G$ is comparable with $L(G)$ remains an open problem even when $H$ is a lateral completion of $G$. The inclusion $L(H) \cap G \supset L(G)$ would follow, for the lateral completion, if we could prove that if $M$ is an $l$-ideal of $G$ the solid hull of $M$ in $H$ (or even in $G(1)$ ) is again an $l$-ideal. We summarise our results as follows.

THEOREM 5.10. If $H$ is the lateral completion of $G$, then

$$
L(G) \cup(L(H) \cap G) \subset D(H) \cap G=D(G) \subset R(H) \cap G=R(G) .
$$

\section{Lattice groups which satisfy condition (F)}

This section is chiefly concerned with providing a simplified proof of Theorem 6.1 in Conrad (1969). We refer to sections 5, 6 of Conrad (1969) for terminology, references and proofs not given here. An element $s$ of a lattice grnup $G$ is called basic if $s>0$ and $\{x \in G: 0 \leqq x \leqq s\}$ is totally ordered. A positive disjoint subset $S$ of $G$ is a basis of $G$ if $S$ is a maximal positive disjoint subset of $G$ and every element of $S$ is basic. If $G$ has a basis $S$, and $H$ is the lateral completion of $G$ it is easily verified that $S$ is also a basis for $H$. 
Suppose now that $G$ is a lattice group which satisfies the condition $(F)$ that each positive element of $G$ exceeds at most a finite number of disjoint elements; i.e. each upper bounded positive disjoint subset is finite. In particular, $G$ has a basis, Conrad (1969), Theorem 5.2, each element of $G^{+}$has only a finite number of values and can be expressed as the supremum of a finite number of disjoint special elements, Conrad (1969), Theorem 2.5, 3.7. If $g \in G, g$ is special if $g$ has precisely one value. Note that if $m, n$ are positive special elements of $G$ and $m \wedge n \neq 0$ then the values of $m$ and $n$ are comparable and there exists a positive integer $p$ such that $m \leqq p n$ or $n \leqq p m$.

We now attack Conrad's Theorem.

THEOREM 6.1. (Conrad (1969), Theorem 6.1). If $G$ is a lattice group which satisfies condition $(F)$ and $H$ is an $\mathscr{L}$-completion of $G$ then, $H=G(1)$,

$$
H^{+}=\{\bigvee M: M \in \mathscr{D}\},
$$

and hence $H$ is the lateral completion of $G$.

(Recall that $\mathscr{D}$ is the set of positive disjoint subsets of $G$.)

Proof. Let $P=\{\bigvee M: M \in \mathscr{D}\}$ and suppose $x=\bigvee M, y=\bigvee N$ with $M, N \in \mathscr{D}$.

$$
(x-y)^{+} \in P \text { and }(-y+x)^{+} \in P .
$$

We have $(x-y)^{+}=\bigvee\{m-m \wedge y: m \in M\}$. Now, $m \wedge y=\bigvee\{m \wedge n$ : $n \in N\}$ and $\{m \wedge n: n \in N\}$ is a disjoint subset of $G$ every element of which is less than or equal to $m$. By condition $(F)$ at most a finite number of the $m \wedge n$ $(n \in N)$ are non-zero. Hence $m \wedge y \in G$ and $\{m-m \wedge y: m \in M\} \in \mathscr{D}$. Thus $(x-y)^{+} \in P$ and similarly $(-y+x)^{+} \in P$.

(2) $x+y \in P$.

Without loss of generality we may assume that all elements of $M$ and $N$ are special. We partition $M$ and $N$ as follows:

$$
\begin{aligned}
& M_{0}=\{m \in M: m \wedge y=0\}, \\
& M_{1}=\{m \in M: \text { for some } n \in N \text { and some integer } p, p m \geqq n\}, \\
& M_{2}=M \sim\left(M_{0} \cup M_{1}\right), \\
& N_{0}=\{n \in N: n \wedge x=0\}, \\
& N_{2}=\{n \in N: \text { for some } m \in M \text { and some integer } p, p m \geqq n\}, \\
& N_{1}=N \sim\left(N_{0} \cup N_{2}\right) .
\end{aligned}
$$

It is readily checked that the suprema of these six sets are pairwise disjoint except for the pairs $\bigvee M_{1}, \bigvee N_{2}$ and $\bigvee M_{2}, \bigvee N_{1}$. Hence

$$
x+y=\left(\bigvee M_{0}\right) \vee\left(\vee M_{1}+\bigvee N_{2}\right) \vee\left(\vee M_{2}+\bigvee N_{1}\right) \vee\left(\vee N_{0}\right),
$$

and it is sufficient to show that $\bigvee M_{1}+\bigvee N_{2} \in P$ and $\bigvee M_{2}+\bigvee N_{1} \in P$. 
For each $m \in M_{1}$ let $N_{2 m}=\left\{n \in N_{2}: m \wedge n \neq 0\right\}$. Then, by condition $(F)$ each $N_{2 m}$ is finite and, by definition, $N_{2}=\bigcup_{m \in M_{1}} N_{2 m}$. Let $n_{m}=\bigvee N_{2 m}$ $\left(m \in M_{1}\right)$, then $n_{m} \in G$ and $n_{m} \leqq p m$ for some integer $p$. It follows that $n_{m} \wedge n_{m^{\prime}}=0$ if $m, m^{\prime} \in M_{1}$ and $m \neq m^{\prime}$.

Thus, $m+n_{m^{\prime}}=m \vee n_{m^{\prime}} \leqq\left(m+n_{m}\right) \vee\left(m^{\prime}+n_{m^{\prime}}\right) ;$ and $\left(m+n_{m}\right) \wedge\left(m^{\prime}+n_{m^{\prime}}\right)$ $=0\left(m, m^{\prime} \in M_{1}, m \neq m^{\prime}\right)$. Hence,

$$
\bigvee M_{1}+\bigvee N_{2}=\bigvee\left\{m+n_{m^{\prime}}: m, m^{\prime} \in M\right\}=\bigvee\left\{m+n_{m}: m \in M\right\} \in P .
$$

It follows similarly that $\bigvee M_{2}+\bigvee N_{1} \in P$ and hence $x+y \in P$.

(3) If $[P]=\{x-y: x, y \in P\}$, then $[P]=G(1)$.

Suppose $u, v \in[P]$, then

$$
(u+v)^{+}=\left(u^{+}-u^{+} \wedge v^{-}\right)+\left(-u^{-} \wedge v^{+}+v^{+}\right) .
$$

By (1), $u^{+}, u^{-}, v^{+}, v^{-} \in P$, then,

$$
\begin{gathered}
u^{+}-u^{+} \wedge v^{-}=\left(u^{+}-v^{-}\right)^{+} \in P, \\
-u^{-} \wedge v^{+}+v^{+}=\left(-u^{-}+v^{+}\right)^{+} \in P,
\end{gathered}
$$

so, by (2), $(u+v)^{+} \in P$. Similarly $(u+v)^{-} \in P$ so $[P]$ is a subgroup of $H$ and, because $P$ generates $G(1),[P] \subset G(1)$.

Also if $u \in[P], u^{+} \in P$, as above, so that $P$ is a sub lattice group of $G(1)$ and hence $P=G(1)$.

It is clear from the proof of (3) that $P=G(1)^{+}$. It follows that $G(1)$ is laterally complete and, hence, that $H=G(1)$ is the lateral completion of $G$.

\section{References}

I. G. Amemiya (1953), 'A general spectral theory in semi-ordered linear spaces', J. Fac. Sci. Hokkaido Univ. Ser. I, 12, 111-156.

S. J. Bernau (1966), 'Orthocompletion of lattice groups', Proc. London Math. Soc. (3) 16, 107 130.

S. J. Bernau (1965), 'Unique representation of Archimedean lattice groups and normal Archimedean lattice rings', Proc. London Math. Soc. (3) 15, 599-631.

R. D. Byrd (1967), 'Complete distributivity in lattice-ordered groups', Pacific J. Math. 20, 423432.

R. D. Byrd and J. T. Lloyd (1969), 'A note on lateral completions in lattice-ordered groups', J. London Math. Soc. (2) 1, 358-362.

R. D. Byrd and J. T. Lloyd (1967), 'Closed subgroups and complete distributivity in latticeordered groups', Math. Zeit. 101, 123-130.

P. F. Conrad, 'Lateral completion of lattice-ordered groups', Proc. London Math. Soc. (3) 19, $444-480$.

P. F. Conrad (1964), 'The relation between the radical of a lattice-orderd group and complete distributivity', Pacific J. Math. 14, 493-499.

P. F. Conrad (1961), 'Some structure theorems for lattice-ordered groups', Trans. Amer. Math. Soc. 99, 212-240. 
P. F. Conrad (1965), 'The lattice of all conve 1-subgroups of a lattice-ordered group', Czech. Math. J. 15, 101-123.

C. Holland (1963), 'The lattice-ordered group of automorphisms of an ordered set', Michigan Math. J. 10, 71-82.

P. Lorenzen (1949), 'Uber halbgeordnete Gruppen', Math. Zeit. 52 484-526.

N. Nakano (1950), Modern Spectarl Theory, (Tokyo 1950).

A. G. Pinkser (1949), 'E tended semiordered groups and spaces', Uchen Zapiski Lemingrad Gos. Ped. Inst. 86, 236-365.

A.I. Veksler and V.A. Geiler (1972), 'Order and disjoint comppeteness of linear partially ordered spaces', Siberian Math. J. 13, 30-35, (Translated from Sibirsk. Math. Z. 13 (1972), 43-51).

University of Texas

Austin, Texas 78712

U. S. A. 